\title{
Information Acquisition in Rumor-Based Bank Runs*
}

\author{
Zhiguo $\mathrm{He}^{\dagger} \quad$ Asaf Manela ${ }^{\ddagger}$
}

November 22, 2011

\begin{abstract}
We study the endogenous information acquisition and withdrawal-redeposit decisions of individual agents when a liquidity event triggers a spreading rumor and therefore exposes a bank to a run. Uncertainty about the bank's liquidity and potential failure motivates agents who hear the rumor to acquire additional information. Although the bank run equilibrium is unique given the additional signal's quality, multiple equilibria emerge with endogenous information acquisition activities. A bank run equilibrium exists when agents aggressively acquire information. We study the threshold parameters that eliminate bank runs. Public provision of solvency information (e.g. stress tests) can eliminate bank runs by indirectly crowding-out individual depositors' effort to acquire liquidity information. However, providing too much information that slightly differentiates competing solvent-but-illiquid banks can result in inefficient runs.
\end{abstract}

JEL Classification: D8, G2

Keywords: Bank runs, learning, information acquisition, asynchronous awareness, temporal coordination, stress tests

${ }^{*}$ Preliminary and Reference Incomplete. We thank Douglas Diamond, Philip Dybvig, Alan Moreira, and Robert Vishny as well as seminar participants at Chicago Booth and Wash U for helpful comments. Part of this project was completed while Zhiguo He visited the Institution of Financial Studies at Southwest University of Finance and Economics in summer 2011.

${ }^{\dagger}$ University of Chicago, Booth School of Business, zhiguo.he@chicagobooth.edu

${ }^{\ddagger}$ Washington University in St. Louis, Olin Business School, amanela@wustl.edu 


\section{Introduction}

Bank runs returned to the headlines during the financial crisis of 2007-2009 with the failures of Northern Rock, IndyMac and other depository institutions. The largest ever failure by bank assets resulted from the run on Washington Mutual (WaMu) in 2008. Figure 1 shows that the run on WaMu was dynamic in its nature: the bank experienced the gradual withdrawal of more than $\$ 16$ billion in the days leading up to its takeover by the FDIC. Moreover, recent empirical work by Iyer and Puri (forthcoming 2011) uses depositor level data to study a solvent Indian bank that survived a run, and their data shows running depositors returning to the surviving bank when they realize the bank will not fail.

Redepositing after running suggests that uncertainty about bank strength plays an important role in bank runs. In this paper, we provide a dynamic bank run model featuring learning and endogenous information acquisition that sheds light on several important questions: Will endogenous information acquisition exacerbate the inefficient bank run equilibrium? What are the policy implications for a social planner aiming to eliminate run equilibria?

Learning and information acquisition are indeed considered some of the key drivers of bank runs, both of which affect government disclosure policy regarding bank regulatory information. One such situation where information is key is at the release of stress test results (Bernanke, 2010). Given the newly found popularity of stress tests both in the U.S. and in Europe, we show that public provision of solvency information can help curb the private information acquisition effort on bank liquidity. As a result, carefully constructed stress tests can help prevent bank runs by crowding out information acquisition by individuals. The planner must be careful however to avoid providing too much information that differentiates competing solvent-but-illiquid banks, for such information can start a run. ${ }^{1}$

We incorporate uncertainty about the bank's liquidity into the asynchronous awareness framework of Abreu and Brunnermeier (2003). In the baseline model, at some random time a liquidity event may occur for a fundamentally solvent bank, so that it becomes illiquid (i.e., limited amount of cash reserves and susceptible to a run) or remains liquid (with sufficient reserves and not subject

\footnotetext{
${ }^{1}$ One recent such case is the Bailout of the Big 9 banks on October 13, 2008, in which Treasury Secretary Paulson forced all banks to accept the bailout package to hide any differences in the strength of the competing banks from depositors.
} 
Figure 1: The Dynamic Nature of the Run on WaMu

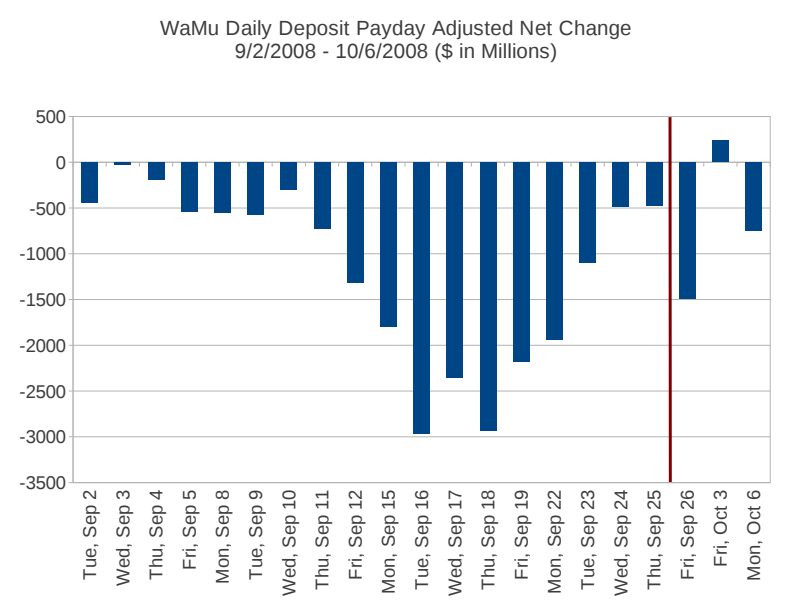

Daily net change in deposits as reported by Washington Mutual Bank to the Office of Thrift Supervision (OTS). We take out Friday and end-of-month fixed effects, i.e. days with automatic payday deposits estimated over the preceding 52 days. The OTS appointed the FDIC as receiver of WaMu on the evening of September 25, 2008 (the vertical line in the figure) which then sold it to JPMorgan Chase.

to run). This triggers the spread of a rumor in the population that exposes the bank to a run. Agents learn about whether the bank is liquid from the passage of time without failure, as well as about the time that the liquidity event started. The redepositing behavior documented by Iyer and Puri (forthcoming 2011) follows naturally from our introduction of uncertainty, as agents who withdraw earlier would redeposit their funds into the bank if the bank turns out to be liquid.

Uncertainty motivates informed agents who hear the rumor to acquire additional information at an endogenously determined signal quality. The realized signal may be utterly uninformative, or reveals the bank liquidity state perfectly. The higher the signal's quality, the more likely is the signal to reveal the bank's liquidity status. If the bank is indeed illiquid, then in aggregate a higher quality translates to a greater (smaller) fraction of agents who (do not) know the bank is illiquid.

The presence of agents with heterogeneous information naturally allows us to derive the unique (responsive) bank run equilibrium as an interior solution, given the signal quality about bank illiquidity. $^{2}$ Conditional on the bank being illiquid, the agents with informative, i.e., "low," signals withdraw immediately due to a high bank failure hazard rate according to their information, while the agents with uninformative, i.e., "medium," signals wait. When all agents with uninformative

\footnotetext{
${ }^{2}$ In Abreu and Brunnermeier (2003), the interior equilibrium is generated under the assumption that the bubble component is exogenously decreasing over time. This time-varying attacking benefit drives arbitragers to attack only when the bubble component drops to certain level.
} 
medium signals wait for a bit longer, they know that there are more agents with informative low signals who have already withdrawn before them. As a result, the bank failure hazard rate endogenously rises because more agents are running earlier. This "fear-of-low-signal-agents" generates a time-varying marginal cost of waiting for agents with the medium signal, which allows us to find the unique endogenous withdrawal time that equates the marginal benefit of waiting.

Given the unique bank run equilibrium, the optimal signal quality for individual agents trades off the convex cost of acquiring it with a greater probability of receiving an informative signal. Moreover, the chosen quality of information affects the equilibrium survival time of the failing bank. This intricate feedback effect between bank runs and information acquisition generally leads to at most two equilibria: one equilibrium where agents do not acquire information and also do not run, while the other equilibrium where agents acquire information aggressively and also run on the illiquid bank.

We perform comparative static analysis focusing on parameters such that the run equilibrium is possible. When each agent finds it easier to acquire information, naturally the quality of information increases. This further leads to a shorter survival period of the illiquid bank, which motivates each agent to learn even more aggressively, and amplifies the initial effect of a lower information cost. ${ }^{3}$

We also decouple the speed by which information spreads from the length of the awareness window over which information spreads. ${ }^{4}$ Contrary to the static intuition, we find that when the awareness window widens so that everybody knows that potentially more agents run on the bank, the illiquid bank survives longer. This counter-intuitive result arises due to the novel uncertainty structure that we introduce in this framework, as the agent who hears the rumor also observes that the bank is still alive. If the awareness window is wide, the rumor could have started spreading a long time before he hears it. Thus, conditional on the bank surviving long enough, the bank is more likely to be liquid, leading to lower running incentives.

Our rich dynamic setting allows us to study the threshold parameters that eliminate bank runs altogether in a nontrivial way. The social planner can, for example, adjust bank reserve require-

\footnotetext{
${ }^{3}$ This effect suggests information acquisition complementarities among depositors. Hellwig and Veldkamp (2009) show that information acquisition exhibits complementarity if and only if the actions are complementary. In our model although running on the bank is complementary, sequential information acquisitions do not necessarily exhibit complementarity.

${ }^{4}$ By contrast, in Abreu and Brunnermeier (2003) the speed by which information spreads is one over the length of the awareness window.
} 
ments in response to heterogeneity in the rumor spreading rate and in the length of the awareness window. In particular, the liquidity requirement is far less than the maximum potential withdrawal, which is the minimum reserve requirement from a static Diamond and Dybvig perspective. Though beyond the scope of the current paper, given the right data, a calibration of our model can certainly result in quantitatively meaningful policy recommendations.

The first extension of our baseline model considers fundamentally insolvent banks. The presence of insolvent banks implies that information might be socially good. However, when agents privately collect information about bank solvency, they inevitably learn about bank liquidity. Although these two layers of information are different, they are inevitably related because effort spent acquiring one reduces the effort needed to acquire the other. The government can provide public information about bank solvency to crowd out the private insolvency information acquisition; now since everyone knows that other agents do not have superior information about bank liquidity, this reduces individual liquidity information acquisition and can potentially eliminate runs on illiquid banks. As we already mentioned, this result has important implications for the amount of information released in stress tests of depository institutions.

A second extension we consider is competition between two solvent but potentially illiquid banks. Instead of holding cash, a bank run in this setting involves the transfer of funds from one bank to another. In a competitive environment, small differences in liquidity can result in runs on slightly weaker banks and their subsequent failure. In this case, the planner would want to inject noise into the system, so that individual agents with less informative signals are more likely to stay in their original bank without knowing which one is more liquid. By forcing all of the "Big 9" banks to take government capital on October 13, 2008, the U.S. government was in fact injecting noise about the liquidity of competing solvent banks into the economy.

Our model belongs to a vast literature on the role of information in bank runs, and it is beyond the scope of our paper to have a thorough review on this topic. ${ }^{5}$ Chari and Jagannathan (1988) shares the same spirit on learning as the cause of panic-based bank runs. In their model, depositors may withdraw early due to individual liquidity reasons as in the classic Diamond and Dybvig (1983), or because they possess superior information about bank assets, and depositors cannot distinguish

\footnotetext{
${ }^{5}$ In early contributions, Gorton (1985) studies suspension of convertibility, Bhattacharya and Gale (1987) focus on information about fraction of early depositors, and Jacklin and Bhattacharya (1988) places the emphasis of comparing equity contracts with deposit contracts.
} 
between them. Since both elements are random, depositors might mistakenly run on the bank even without bad news about bank assets. In a recent paper, Gu (2011) subdivides the interim period of the static Diamond and Dybvig model into $N$ periods and allows depositors to choose their withdrawal time. Herding arises because depositors observe other depositors actions in addition to a noisy private signal about the quality the bank. By contrast, in our model, synchronization risk arises because agents cannot observe a line forming in front of the bank as one might expect in an electronic age. Based on the Morris and Shin (2002) global games technique, Goldstein and Pauzner (2005) study the optimal deposit contract by deriving a unique equilibrium when depositors in a Diamond and Dybvig type setting are endowed with private noisy signals about bank fundamentals. We allow for endogenous information acquisition, and show that excessive socially wasteful learning may lead to socially inefficient runs on solvent-but-illiquid banks. ${ }^{6}$

Our paper contributes to a recent theoretical literature that studies dynamic bank runs. Wallace (1988) and Green and Lin (2003) are earlier papers that study the sequential service constraint in the Diamond and Dybvig model. Gu (2011) is a dynamic model in nature, that studies depositors' withdrawal strategy sequentially. He and Xiong (forthcoming 2011a) develop a continuous-time debt run model of a firm with a time-varying fundamental and a staggered debt structure. Similar to the asynchronous timing structure employed in our paper, each creditor in He and Xiong coordinates his rollover decision with future maturing creditors. ${ }^{7}$ We emphasize the role that information and learning play in runs, and how the government can eliminate runs when individuals acquire information.

Abreu and Brunnermeier $(2002,2003)$ consider the asynchronous timing of awareness to study bubbles. In their model, delayed arbitrage is rational because agents do not know whether other agents are aware of this arbitrage opportunity, and more importantly, when they are taking action on it. ${ }^{8}$ We add to their model uncertainty about the capacity of the bubble (bank), allow agents to acquire additional information upon awareness, and decouple the spreading rate from the length

\footnotetext{
${ }^{6}$ This result is related to Hellwig and Veldkamp (2009) who show that in the Morris and Shin (2002) beauty contest framework, complementarity in actions leads to complementarity in information acquisition, therefore potential multiplicity of equilibria. However, their setting is different from ours. In fact, as we shown in Section 4.4, substitutability naturally arises in our endogenous dynamic learning framework.

${ }^{7}$ In other related papers, Acharya, Gale, and Yorulmazer (2011) shows the rollover of short-term debt may reduce debt capacity to zero, and He and Xiong (forthcoming 2011b) analyze the role played by market illiquidity, through the rollover channel, in exacerbating the conflict between debt and equity holders.

${ }^{8}$ Brunnermeier and Morgan (2010) generalize this idea to a class of "clock games" and test its main predictions in controlled experiments.
} 
of the awareness window.

The paper proceeds as follows. Section 2 describes the setting and solves the agent's learning problem. Section 3 characterizes the individual agent's optimal withdrawal policy, and Section 4 analyzes the bank run equilibrium with information acquisition. Section 5 considers extensions, and we conclude in Section 6. Proofs are in the Appendix.

\section{The Model}

We first describe the economy and the individual agent's problem in our model. We then characterize the agent's belief updating, which is crucial in studying the individual agent's optimal strategy.

\subsection{The Setting}

\subsubsection{Technology}

Time is continuous on $t \in[0, \infty)$. A continuum of risk-neutral agents (depositors) with unit mass have an infinite horizon and maximize their expected utility from consumption with a zero discount rate.

Bank deposits yield a constant rate of return $r>0$ when the bank is operating, while holding cash outside the bank earns zero return. Broadly, one can interpret the bank as some investment vehicles in the shadow banking system or even the entire financial system, and the positive relative wedge $r>0$ reflects either a higher investment growth rate or a convenience yield for keeping funds in the institution. To avoid exploding values, we assume that the bank's growth stops at some "maturing" event modeled as a Poisson shock with intensity $\delta>r$. Following this public event, the growth-less bank liquidates without loss, and the game ends in the sense that each agent gets his deposit back for consumption. Throughout, this maturing event will be independent of any other random variables that we consider. ${ }^{9}$

\footnotetext{
${ }^{9}$ This assumption plays no role in our analysis except making the value of the one dollar inside the bank finite (as the liquid bank will grow always). Alternatively, we could assume that each individual agent suffers liquidity shocks that require immediate consumption (therefore withdrawal). Unfortunately, this brings the troublesome issue of agent replacement.
} 


\subsubsection{Uncertainty about Bank Liquidity}

In our model there are two potential types of banks that are fundamentally solvent, with one type of bank being "illiquid," and a second "liquid" bank impervious to runs. The uncertainty is crucial to our analysis. Later we will introduce insolvent banks as an extension.

Throughout, bank liquidity is defined as the amount of depositors that it takes to run down the bank. For simplicity, we assume a binary structure for the state of bank liquidity $\widetilde{\kappa}$. For the liquid bank, $\widetilde{\kappa}=\kappa_{H}>1$, i.e., the bank can survive any severe run. However, when the bank is illiquid, $\widetilde{\kappa}$ takes a lower value $\kappa_{L}$ below 1 , and the bank is potentially subject to runs. In other words, the illiquid bank fails when more than a $\kappa$ measure of the depositors have fully withdrawn their funds. One can literally interpret $\widetilde{\kappa}$ as the bank's cash reserves to meet withdrawals; in this paper we broadly interpret $\widetilde{\kappa}$ as the liquidity of the bank.

In our economy, upon awareness, informed agents begin with a prior that $\operatorname{Pr}\left\{\widetilde{\kappa}=\kappa_{L}\right\}=p_{0} \in$ $(0,1)$. When the illiquid bank fails, all remaining depositors in the failed bank recover a fraction $\gamma \in(0,1)$ of their deposits. Afterwards, all agents go to autarky consuming their remaining wealth.

\subsubsection{Liquidity Event and Spreading Rumors}

At $t=0$ the fundamentally solvent bank is liquid, i.e., $\widetilde{\kappa}=\kappa_{H}$. At some unobservable random time $\widetilde{t_{0}}>0$ which we refer to as the liquidity event, the solvent bank may become illiquid, and the uncertainty is as modeled in Section 2.1.2.

Moreover, this liquidity event triggers information to spread in the population. We call those agents who have heard the information as "informed," and those who have not as "uninformed." Since at $t=0$ the bank is liquid, we assume that the agents' beliefs are such that they expect to hold money in the bank unless they hear the information that the bank might be illiquid.

Although the exact liquidity event time $t_{0}$ is publicly unobservable, it is common knowledge that $\widetilde{t_{0}}$ is exponentially distributed on $[0, \infty)$ with cumulative distribution function $\Phi\left(t_{0}\right) \equiv 1-e^{-\theta t_{0}}$, and density $\phi\left(t_{0}\right) \equiv \theta e^{-\theta t_{0}}$. This information structure is meant to capture the essence of an unverified rumor of uncertain origin that spreads gradually in the depositor population. Later on we will call this information about bank's liquidity status a "rumor."

Given a realization of $\widetilde{t_{0}}=t_{0}$, the rumor begins to spread over an interval $\left[t_{0}, t_{0}+\eta\right]$ with a 
positive constant (exogenous) length $\eta$. Following Abreu and Brunnermeier (2003) we refer to $\eta$ as the "awareness window." At any interval $d t$ where $t \in\left(t_{0}, t_{0}+\eta\right)$, uninformed agents become informed by receiving this signal with a probability of $\beta d t$, and this information shock is i.i.d. across the population of uninformed agents. To make the problem interesting, we assume that at time $t_{0}+\eta$ the fraction of informed agents will be sufficient to take down the bank, if they decide to do so.

This information spreading technology is different from that of Abreu and Brunnermeier (2003) in that we allow for separation between the spreading rate $\beta$ and the awareness window $\eta$. Abreu and Brunnermeier assume a linear spreading technology with rate $\frac{1}{\eta}$ so that the entire population becomes informed at $t_{0}+\eta$. This way, the awareness window $\eta$ is artificially tied to the spreading rate $\frac{1}{\eta}$. As we show later, because of the endogenous learning effect, the awareness window $\eta$ has interesting effects opposite to common wisdom.

Given $t_{0}$ and some future time $t \in\left[t_{0}, t_{0}+\eta\right]$, denote by $U\left(t \mid t_{0}\right) \in[0,1]$ the measure of uninformed agents who have not heard the rumor yet. At $t=t_{0}$, every agent is uninformed, so that $U\left(t_{0} \mid t_{0}\right)=1$. The population of uninformed agents evolves as $d U\left(t \mid t_{0}\right)=-\beta U\left(t \mid t_{0}\right) d t$, which implies that

$$
U\left(t \mid t_{0}\right)=e^{-\beta\left(t-t_{0}\right)} \text { for } t \in\left[t_{0}, t_{0}+\eta\right]
$$

and the mass of newly informed agents within $[t, t+d t]$ is

$$
\beta U\left(t \mid t_{0}\right) d t=\beta e^{-\beta\left(t-t_{0}\right)} d t \text { for } t \in\left[t_{0}, t_{0}+\eta\right]
$$

\subsubsection{Information Acquisition}

At time $t_{i}$ when the agent hears the rumor, he can acquire additional information about the bank's type at some convex cost. Specifically, the agent makes an endogenous choice of information quality $q \in[0,1]$ at the cost $\chi(q)=\alpha q^{2} / 2$, where $\alpha>0$ is a positive constant. For tractability, we assume that $\chi(q)$ is the per dollar information cost, so that informed agents face the same problem when they are informed at different times. 
Figure 2: Probability Distribution of the Additional Signal $\tilde{y}$ with Quality $q$

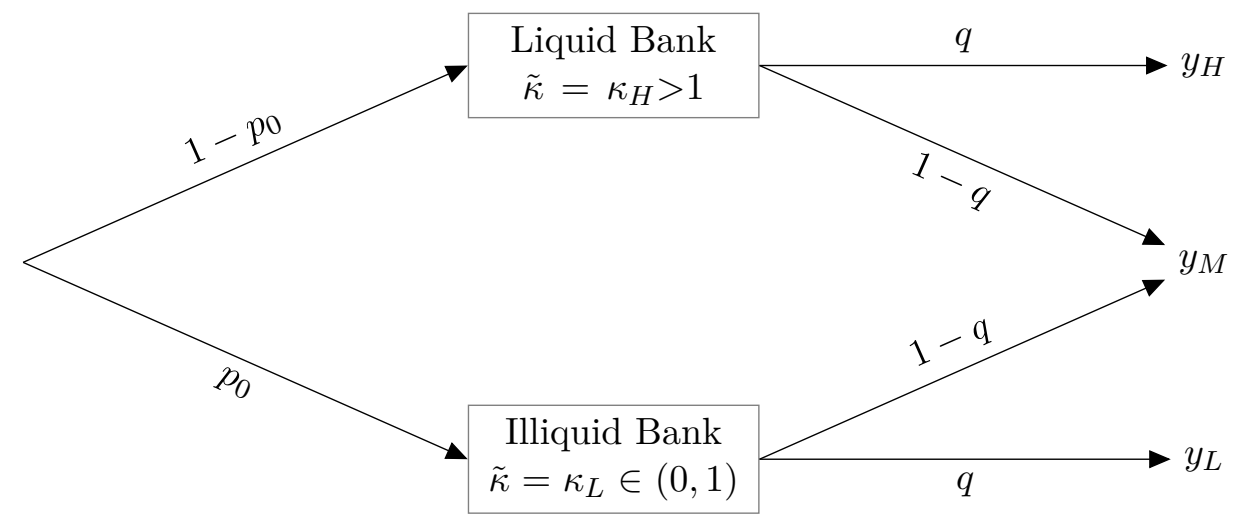

This additional signal takes three possible values $y \in\left\{y_{L}, y_{M}, y_{H}\right\}$ with conditional probabilities:

$$
\begin{aligned}
& \operatorname{Pr}\left\{y=y_{H} \mid \tilde{\kappa}=\kappa_{H}\right\}=q, \operatorname{Pr}\left\{y=y_{M} \mid \tilde{\kappa}=\kappa_{H}\right\}=1-q, \text { and } \operatorname{Pr}\left\{y=y_{L} \mid \tilde{\kappa}=\kappa_{H}\right\}=0 \\
& \operatorname{Pr}\left\{y=y_{L} \mid \tilde{\kappa}=\kappa_{L}\right\}=q, \operatorname{Pr}\left\{y=y_{M} \mid \tilde{\kappa}=\kappa_{L}\right\}=1-q, \text { and } \operatorname{Pr}\left\{y=y_{H} \mid \tilde{\kappa}=\kappa_{L}\right\}=0 .
\end{aligned}
$$

Figure 2 summarizes this distribution. With probability $q$, the bank's liquidity is perfectly revealed by the signal $y_{H}\left(y_{L}\right)$. With probability $1-q$, the agent does not learn anything by receiving the medium signal $y_{M} \cdot{ }^{10}$ The realizations of these signals are i.i.d. across agents, conditional on the underlying state.

\subsubsection{Information Structure}

Call the agent who is informed at $t_{i} \geq t_{0}$ simply agent $t_{i}$. Denote by $\mathcal{F}_{t}^{t_{i}}$ the information set of agent $t_{i}$ at $t>t_{i}$, and let $\mathbf{1}_{t}^{B F} \in\{1,0\}$ indicate whether the bank has failed or not by time $t$. The agent $t_{i}$ 's information set is $\mathcal{F}_{t}^{t_{i}}=\left\{t, t_{i}, \widetilde{y}_{t_{i}}, \mathbf{1}_{t}^{B F}\right\}$, i.e. the informed agent knows the current time, the time that he hears the rumor, the additional signal that he acquired, and whether the bank has failed or not. Recall that the liquidity event $t_{0}$ is not public information.

\footnotetext{
${ }^{10}$ Upon receiving $y_{M}$, the posterior probability of the bank being illiquid remains $\frac{p_{0}(1-q)}{p_{0}(1-q)+\left(1-p_{0}\right)(1-q)}=p_{0}$.
} 


\subsubsection{Agent's Problem}

Now we summarize the problem of an informed agent $t_{i}$ with deposits inside the bank. He will acquire an additional signal $\widetilde{y} \in\left\{y_{L}, y_{M}, y_{H}\right\}$. Based on this information, he can withdraw his deposits whenever he believes bank failure is imminent, and redeposit this cash in the future if the bank's survival sufficiently improves his posterior belief about bank liquidity. To eliminate strategies with infinite transactions, we assume a constant (proportional) transaction cost $k$ per dollar of deposits when the agent (re)deposits his cash into the bank.

The risk neutrality and the bank's superior investment technology imply that it is optimal for the agent to consume only at the bank's exogenous maturing event, or when the bank endogenously failed due to runs. Also, the linearity of this problem implies that a "bang-bang" strategy, i.e. keeping the entire wealth either in or out of the bank, is optimal.

\subsection{Learning}

In our model informed agents need to assess the posterior probability of bank failure based on their information set. To this end we derive the bank failure hazard rate in this section, which is important for later analysis.

\subsubsection{Posterior Belief about $t_{0}$}

The agent $t_{i}$ updates the posterior distribution of $t_{0}$ conditional on his hearing the rumor at $t_{i}$. Given $t_{0}$, for an individual uninformed agent the probability of getting informed over $\left[t_{i}, t_{i}+d t\right]$ is determined as follows. For $t_{i} \in\left[t_{0}, t_{0}+\eta\right)$, the probability of becoming informed at $\left[t_{i}, t_{i}+d t\right]$ but not before $t_{i}$ is $f\left(t_{i} \mid t_{0}\right) \equiv \beta U\left(t_{i} \mid t_{0}\right) d t=\beta e^{-\beta\left(t_{i}-t_{0}\right)} d t$. And, since the rumor stops spreading after $t_{0}+\eta$, we have

$$
f\left(t_{i} \mid t_{0}\right)=\left\{\begin{array}{cc}
\beta e^{-\beta\left(t_{i}-t_{0}\right)} d t & \text { if } t_{i} \in\left[t_{0}, t_{0}+\eta\right), \\
0 & \text { if } t_{i} \geq t_{0}+\eta
\end{array} .\right.
$$

Combining with the density of $\phi\left(t_{0}\right)$, the informed agent $t_{i}$ updates his posterior distribution about the liquidity event timing $t_{0}$.

Similar to Abreu and Brunnermeier (2003) we focus on realizations of $t_{0} \geq \eta$ such that the economy is already in this stationary phase; as shown shortly agent $t_{i}$ 's equilibrium strategy will 
be independent of the exact timing of being informed. ${ }^{11}$

With $t_{0} \geq \eta$, the informed agent $t_{i} \geq t_{0} \geq \eta$ will update his posterior belief about $t_{0}$ as

$$
\phi\left(t_{0} \mid t_{i}\right) \equiv \frac{f\left(t_{i} \mid t_{0}\right) \phi\left(t_{0}\right)}{\int_{t_{i}-\eta}^{t_{i}} f\left(t_{i} \mid s\right) \phi(s) d s}=\frac{\beta e^{-\beta\left(t_{i}-t_{0}\right)} \theta e^{-\theta t_{0}}}{\int_{t_{i}-\eta}^{t_{i}} \beta e^{-\beta\left(t_{i}-s\right)} \theta e^{-\theta s} d s}=\frac{\theta-\beta}{e^{(\theta-\beta) \eta}-1} e^{(\theta-\beta)\left(t_{i}-t_{0}\right)} .
$$

Define $\lambda \equiv \theta-\beta>0$ so that the liquidity event intensity is greater than the rumor spreading rate. $^{12}$ Then

$$
\phi\left(t_{0} \mid t_{i}\right)=\frac{\lambda e^{\lambda\left(t_{i}-t_{0}\right)}}{e^{\lambda \eta}-1}
$$

Integrating (4) over $t_{0}$ we get the conditional distribution function for $t_{0}$ :

$$
\Phi\left(t \mid t_{i}\right) \equiv \operatorname{Pr}\left(\widetilde{t_{0}} \leq t \mid t_{i}\right)=\int_{t_{i}-\eta}^{t} \phi\left(s \mid t_{i}\right) d s= \begin{cases}0 & t<t_{i}-\eta \\ \frac{e^{\lambda \eta}-e^{\lambda\left(t_{i}-t\right)}}{e^{\lambda \eta}-1} & t_{i}-\eta \leq t \leq t_{i} \\ 1 & t>t_{i}\end{cases}
$$

\subsubsection{Bank Failure Hazard Rate}

Suppose that in a symmetric equilibrium, agents believe that the illiquid bank fails at $\widetilde{t_{0}}+\zeta$ where $\zeta$ is a constant to be determined in equilibrium (potentially infinite). Let $F\left(t_{0}\right) \equiv t_{0}+\zeta$ denote the failure time for a given realization of $t_{0}$, conditional on the bank being illiquid, i.e., $\kappa=\kappa_{L}$. Obviously, the event of bank failure is $\left\{F\left(t_{0}\right)<t, \kappa_{L}\right\}$. Moreover, if the bank fails at $t$, then the inferred $t_{0}$ is

$$
t_{0}=F^{-1}(t)=t-\zeta
$$

Denote by $p\left(t \mid t_{i}\right) \equiv \operatorname{Pr}\left\{\widetilde{\kappa}=\kappa_{L} \mid \mathcal{F}_{t}^{t_{i}}\right\}$ the posterior probability at time $t$ for the bank being illiquid. Trivially, bank failure at $t$ reveals that $p\left(t \mid t_{i}\right)=1$. Also given $y_{L}$ or $y_{H}$ signals, $p\left(t \mid t_{i}\right)=1$ or 0 . For agent $t_{i}$ with $y_{M}$ signal, if the bank has not failed at $t$ (i.e. $t<F\left(t_{0}\right)$ ), then his posterior

\footnotetext{
${ }^{11}$ This implies that the economy is stationary. Note that the finite awareness window $\eta$ over which the rumor spreads makes the cases of $t_{0}<\eta$ and $t_{0} \geq \eta$ different. In the event of $t_{0} \geq \eta$, it always holds that $t_{i} \geq \eta$, and rational agents know that $t_{0} \in\left[t_{i}-\eta, t_{i}\right]$. In Appendix B we consider the equilibrium behavior for $t_{0}<\eta$.

${ }^{12}$ The assumption of $\lambda>0$ is for exposition purpose, and the analysis goes though if $\lambda<0$. To see this, if $\lambda<0$, then the conditional density $\phi\left(t_{0} \mid t_{i}\right)$ has to be written as $\frac{(-\lambda) e^{\lambda\left(t_{i}-t_{0}\right)}}{1-e^{\lambda \eta}}$ which is still positive.
} 
belief of bank being illiquid is (recall that $\widetilde{\kappa}$ is independent of $t_{0}$ ):

$$
\begin{aligned}
p\left(t \mid t_{i}\right) & =\operatorname{Pr}\left\{\kappa_{L} \mid t<F\left(t_{0}\right), t_{i}\right\}=\frac{\operatorname{Pr}\left\{t<F\left(t_{0}\right) \mid \kappa_{L}, t_{i}\right\} \operatorname{Pr}\left\{\kappa_{L}\right\}}{\operatorname{Pr}\left\{t<F\left(t_{0}\right) \mid \kappa_{L}, t_{i}\right\} \operatorname{Pr}\left(\kappa_{L}\right)+\operatorname{Pr}\left\{\kappa_{H}\right\}} \\
& =\frac{\left[1-\Phi\left(F^{-1}(t) \mid t_{i}\right)\right] p_{0}}{\left[1-\Phi\left(F^{-1}(t) \mid t_{i}\right)\right] p_{0}+1-p_{0}},
\end{aligned}
$$

We then use (5) and (6) to derive $p\left(t \mid t_{i}\right)$ in closed form. Moreover, as shown later in Section 4.2, in equilibrium if $\zeta$ is finite, then we must have $\zeta \leq \eta$. In this case, when agent $t_{i}$ hears the rumor, his posterior probability of the bank being illiquid is

$$
p\left(t_{i} \mid t_{i}\right)=\frac{\left(e^{\lambda \zeta}-1\right) p_{0}}{\left(1-p_{0}\right)\left(e^{\lambda \eta}-1\right)+\left(e^{\lambda \zeta}-1\right) p_{0}} .
$$

The cumulative distribution of bank failure times $t_{i}+\tau$, conditional on hearing the rumor at $t_{i}$ and the bank has not failed at $t_{i}$, can be derived as

$\Pi\left(t_{i}+\tau \mid t_{i}\right) \equiv p\left(t_{i} \mid t_{i}\right) \operatorname{Pr}\left\{t_{i}<F\left(t_{0}\right) \leq t_{i}+\tau \mid t_{i}, \kappa_{L}\right\}=p\left(t_{i} \mid t_{i}\right) \frac{\Phi\left(F^{-1}\left(t_{i}+\tau\right) \mid t_{i}\right)-\Phi\left(F^{-1}\left(t_{i}\right) \mid t_{i}\right)}{1-\Phi\left(F^{-1}\left(t_{i}\right) \mid t_{i}\right)}$.

By calculating the density $\pi\left(t_{i}+\tau \mid t_{i}\right) \equiv \frac{d \Pi\left(t_{i}+\tau \mid t_{i}\right)}{d \tau}$, we derive the bank failure hazard rate from the perspective of the informed agent $t_{i}$ as

$$
h\left(t_{i}+\tau \mid t_{i}\right) \equiv \frac{\pi\left(t_{i}+\tau \mid t_{i}\right)}{1-\Pi\left(t_{i}+\tau \mid t_{i}\right)}
$$

We present the closed form expression for $h\left(t_{i}+\tau \mid t_{i}\right)$ in the following proposition, and in the proof (placed in Appendix) readers can find expressions for $\Pi\left(t_{i}+\tau \mid t_{i}\right)$ and $\pi\left(t_{i}+\tau \mid t_{i}\right)$.

Proposition 1. Suppose that the illiquid bank fails at $t_{0}+\zeta$ where $\zeta \leq \eta$ which holds in equilibrium. Then the bank failure hazard rate is

$$
h(\tau) \equiv h\left(t_{i}+\tau \mid t_{i}\right)=\frac{\lambda e^{\lambda(\zeta-\tau)} p_{0}}{\left(1-p_{0}\right)\left(e^{\lambda \eta}-1\right)+\left(e^{\lambda(\zeta-\tau)}-1\right) p_{0}} \text { for } \tau \in[0, \zeta]
$$

For $\tau>\zeta, h(\tau)=0$ as the bank is revealed to be liquid.

We have three noteworthy observations. First, the hazard rate in (9) is independent of the absolute timing of agent $t_{i}$ becoming informed, therefore we can denote $h\left(t_{i}+\tau \mid t_{i}\right)$ by $h(\tau)$. This 
property guarantees the stationarity of our model.

Second, the hazard rate only depends on the remaining survival time from the potential bank failure time, i.e., $\zeta-\tau$. This is intuitive: because the agents in our economy are uncertain about the exact timing of liquidity event $t_{0}$ that the rumor starts to spread, from the perspective of the agents the bank failure hazard rate will depend on how far the economy is away from the maximum potential failure time $t_{0}+\zeta$. The equilibrium remaining survival time is important for our later analysis.

Third, the above analysis is carried out for $y_{M}$ agents who remain uncertain about the bank's type. The analysis for $y_{L}\left(y_{H}\right)$ agents are straightforward by simply replacing $p_{0}$ with $1(0)$ in equation (9).

\subsection{Parameter Restrictions}

We impose the following parametrization condition throughout the paper:

$$
\frac{p_{0}}{1-p_{0}}>e^{\lambda \eta}-1
$$

This condition implies that the bank hazard rate given in Proposition 1 is increasing with $\tau$, i.e., the time that elapsed since hearing the rumor. Equivalently, this implies that the bank failure hazard rate is decreasing in the remaining survival time $\zeta-\tau$.

Second, since the upper bound of the measure of informed agent is $1-U\left(t_{0}+\eta \mid t_{0}\right)=1-e^{-\beta \eta}$, for the model to be interesting we require that the illiquid bank can potentially fail if all informed agents run immediately, i.e.,

$$
1-e^{-\beta \eta}>\kappa_{L}
$$

Third, we focus on the case where both $y_{M}$ agents and $y_{L}$ agents are driving the bank failure. Conditional on the bank being illiquid, there will be $q$ measure of $y_{L}$ agents. Therefore, instead of $q \in[0,1]$, we further impose that the signal quality has to satisfy

$$
q \in\left[0, \frac{\kappa_{L}}{1-e^{-\beta \eta}}\right]
$$

Under this condition, because signal quality $q<\frac{\kappa_{L}}{1-e^{-\beta \eta}}$, agents with $y_{L}$ signal alone are not enough 
to take down the bank.

Fourth, we require that the maturity shock intensity $\delta$ is moderate so that

$$
\frac{\delta\left(1-p_{0}\right)\left(e^{\lambda \eta}-1\right) \frac{r(r-k \delta)}{\delta-r}}{\lambda(r-\lambda(1-\gamma)) p_{0}} \in(0,1)
$$

Note that this requirement implies that $r-\lambda(1-\gamma)>0$. The only purpose of this condition is to guarantee the optimality of thresholds strategy even if the bank randomly matures.

Finally, we assume that

$$
(\lambda(1-\gamma)-r) e^{\lambda \eta}+r>0
$$

as we will see in Section 3.3, this implies that the agent with $y_{L}$ signal withdraw immediately, if the bank run equilibrium exists.

\section{Optimal Withdrawal Strategies}

In this section, we present the key proposition that characterizes the individual agent's optimal withdrawal policy, taking both the equilibrium bank survival time $\zeta$ and information quality $q$ as given.

\subsection{Hamilton-Jacobi-Bellman (HJB) Equations}

Denote by $V_{I}\left(\tau ; t_{i}\right)$ the agent's value of one dollar inside the bank at time $t_{i}+\tau$, where $\tau \geq 0$ is the time elapsed since agent $t_{i}$ heard the rumor. Due to stationarity, $V_{I}\left(\tau ; t_{i}\right)$ is a function of $\tau$ only and we suppress the argument $t_{i}$ wherever appropriate. Similarly, denote by $V_{O}\left(\tau ; t_{i}\right)=V_{O}(\tau)$ the value of one dollar outside of the bank at time $t_{i}+\tau$. Because withdrawal involves no transaction cost while redepositing costs $k, V_{I}(\tau) \geq V_{O}(\tau)$ and $V_{O}(\tau) \geq(1-k) V_{I}(\tau)$ for all $\tau \geq 0$.

When $\tau \geq \zeta$, the surviving bank is for sure safe. One dollar inside the bank will grow at $r$ until the maturing event (occurs with Poisson intensity $\delta$ ), which has a value of

$$
V_{I}(\tau)=\int_{0}^{\infty} e^{r s} \delta e^{-\delta s} d s=\frac{\delta}{\delta-r} \text { for } \tau \geq \zeta
$$

The value of one dollar outside the bank is $V_{O}(\zeta)=(1-k) V_{I}(\zeta)=\frac{(1-k) \delta}{\delta-r}>1$, which holds when 
$k$ is sufficiently small.

For $\tau<\zeta$ the agent has not fully been assured that the bank is liquid, and consider a dollar outside the bank. At any point in time, if the status-quo position (i.e. keeping the dollar outside the bank) is optimal, then the following HJB equation must hold:

$$
0 \underset{\text { Bank failure }}{0} \underset{\text { Bank matures }}{\left(1-V_{O}(\tau)\right)}+\underset{\text { Time change }}{V_{O}^{\prime}(\tau)}
$$

Here, the first term is the impact of bank failure: with hazard rate $h(\tau)$ the bank fails so that the agent receives 1 and loses his value $V_{O}(\tau) \cdot{ }^{13}$ The second term captures the bank asset maturing event, and the third term is the change due to time elapse. Combined with the option of redepositing immediately (with transaction cost $k$ ), the HJB equation for one-dollar outside the bank is

$$
0=\max \left\{\underset{\text { Bank failure }}{h(\tau)\left(1-V_{O}(\tau)\right)+\underset{\text { Bank matures }}{\delta\left(1-V_{O}(\tau)\right)}+\underset{\text { Time change }}{V_{O}^{\prime}(\tau)},(1-k) V_{I}(\tau)-V_{O}(\tau)}\right\}
$$

Similarly, for a dollar inside the bank, its value $V_{I}(\tau)$ must satisfy the following HJB equation:

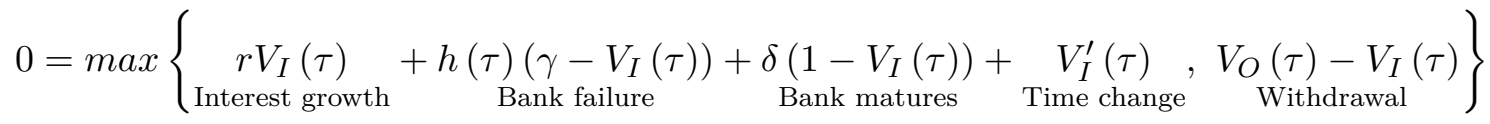

\subsection{Optimal Strategies}

We solve the ordinary differential equation (16) in the interval $[\tau, \zeta]$ with the boundary condition $\widehat{V}_{O}(\zeta)=V_{O}(\zeta)$, and denote its solution as $\widehat{V}_{O}(\tau)$. It admits the following closed-form solution:

$$
\widehat{V}_{O}(\tau)=\frac{e^{\lambda \eta}\left(1-p_{0}\right)-1+e^{\lambda(\zeta-\tau)} p_{0}+e^{-\delta(\zeta-\tau)}\left(1-p_{0}\right)\left(e^{\lambda \eta}-1\right) \frac{r-k \delta}{\delta-r}}{\left(1-p_{0}\right)\left(e^{\lambda \eta}-1\right)+\left(e^{\lambda(\zeta-\tau)}-1\right) p_{0}}
$$

which allows us to characterize the agent's optimal strategy parsimoniously. In general $V_{O}(\tau) \geq$ $\widehat{V}_{O}(\tau): \widehat{V}_{O}(\tau)$ is the value at time $\tau$ by simply staying outside the bank until $\zeta$ (and redepositing at $\zeta^{+}$if the bank is liquid and survives the run), and a priori this simple continuation strategy may not be optimal.

Define the following function $g(\tau)$ which captures the first-order impact of the withdrawal

\footnotetext{
${ }^{13}$ The analysis holds for all signals as Proposition 1 gives $h(\tau)$ as a function of $p_{0}$.
} 
decision:

$$
g(\tau) \equiv h(\tau)(1-\gamma)-r \widehat{V}_{O}(\tau)
$$

Here, $g(\tau)$ is difference between the instantaneous loss from one dollar due to potential bank failure (i.e. $h(\tau)(1-\gamma)$ ), and $r \widehat{V}_{O}(\tau)$ which is the instantaneous return of taking one dollar out now, keeping outside the bank until $\zeta$, and redepositing back if the bank survives. The underlying assumption here is that a threshold strategy is optimal (i.e. redepositing is never optimal before $\zeta)$ which is verified in Proposition 2.

At the optimal withdrawal time $\tau_{w}$, we have the first-order condition $g\left(\tau_{w}\right)=0$, i.e.,

$$
h\left(\tau_{w}\right)(1-\gamma)=r \widehat{V}_{O}\left(\tau_{w}\right)=r\left[1+\left(1-p\left(t_{i}+\tau_{w} \mid t_{i}\right)\right) e^{-\delta\left(\zeta-\tau_{w}\right)} \frac{r-k \delta}{\delta-r}\right]
$$

where we have used (19) and (7) in rewriting this intuitive expression. As mentioned above, the left hand side captures the marginal cost of staying, which is the hazard rate multiplying the bank failure loss. The right hand side captures the marginal benefit of staying, which is the growth rate $r$ multiplying the agent's continuation value of one dollar by withdrawing and redepositing after $\zeta$. For the total continuation value in the bracket of right hand side in (21), the first term 1 is the principal amount which is present in Abreu and Brunnermeier (2003). The second term consists of the option value of future redepositing. Here, $1-p\left(t_{i}+\tau_{w} \mid t_{i}\right)$ is the probability of the bank being liquid (and surviving eventually) conditional on bank survival at $\tau_{w}, e^{-\delta\left(\zeta-\tau_{w}\right)}$ is the discounting, and finally

$$
\frac{r-k \delta}{\delta-r}=V_{O}(\zeta)-1=\frac{(1-k) \delta}{\delta-r}-1>0
$$

is the additional payoff from redepositing. Apparently, when $p_{0}=1$ so that there is no uncertainty about bank liquidity, this option value term vanishes.

The next proposition shows formally that a threshold strategy is optimal based on the function $g(\tau)$

Proposition 2. Given the equilibrium bank survival time $\zeta$, the optimal policy for the agent with $y_{M}$ is as follows:

1. If $g(\zeta) \leq 0$, then it is optimal to stay in the bank always. 
2. If $g(0) \geq 0$, then it is optimal to withdraw at 0 and redeposit right after $\zeta$.

3. Otherwise, we must have $g(0)<0$ and $g(\zeta)>0$, and there exists a unique waiting time $\tau_{w} \in(0, \zeta)$ so that $g\left(\tau_{w}\right)=0$, and withdrawing at $\tau_{w}$ and redepositing at $\zeta^{+}$is optimal.

\subsection{Value Functions}

In this section we derive the agent's value as a function of signals. We have studied the optimal strategy for the agent with $y_{M}$ signal. It is clear that the agents with high signal $y_{H}$ should keep their deposits in the bank always. And, for agents who receive low signals, if

$$
g\left(0 ; p_{0}=1\right)=\frac{(\lambda(1-\gamma)-r) e^{\lambda \zeta}+r}{e^{\lambda \zeta}-1}>0
$$

then it is optimal to withdraw immediately. Because $\zeta<\eta$ and $\lambda(1-\gamma)<r$, (22) is implied by parameter condition (14). In fact, we will show later in Section 4.2 that condition (22) has to hold for any interior bank run equilibria. In our model, if $y_{L}$ agents want to wait some positive amount of time, then generically bank run equilibria do not exist.

Proposition 3. Suppose that $g(0)<0$ and $g(\zeta)>0$ so that the agents with $y_{M}$ signals are waiting $\tau_{w}$ given in (21). Then upon hearing the rumor, the values conditional on signals are

$$
\begin{aligned}
V_{I}\left(0 \mid y_{L}\right) & =1, V_{I}\left(0 \mid y_{H}\right)=\frac{\delta}{\delta-r} \\
V_{I}\left(0 \mid y_{M}\right) & =\frac{\frac{\delta\left(e^{\lambda \eta}\left(1-p_{0}\right)-1\right)}{\delta-r}\left(1-e^{-(\delta-r) \tau_{w}}\right)+\frac{\delta+\lambda \gamma}{\lambda+\delta-r} e^{\lambda \zeta} p_{0}+e^{-(\delta-r) \tau_{w}} e^{\lambda\left(\zeta-\tau_{w}\right)} p_{0}\left(\frac{(\lambda+\delta)(\lambda(1-\gamma)-r)}{r(\lambda+\delta-r)}\right)}{e^{\lambda \eta}-1-e^{\lambda \eta} p_{0}+e^{\lambda \zeta} p_{0}}
\end{aligned}
$$

where $\tau_{w}$ satisfies the first-order condition in (21).

\section{Bank Run Equilibrium}

\subsection{Cumulative Withdrawals Given $q$}

In our model, the illiquid bank fails at $t_{0}+\zeta$ so that aggregate cumulative withdrawals by informed agents deplete the illiquid bank's capacity $\kappa_{L}$. Two groups of informed agents withdraw from the illiquid bank. The first group is $y_{L}$ agents with $q$ measure in aggregate per unit of time. Therefore 
Figure 3: Cumulative Withdrawals
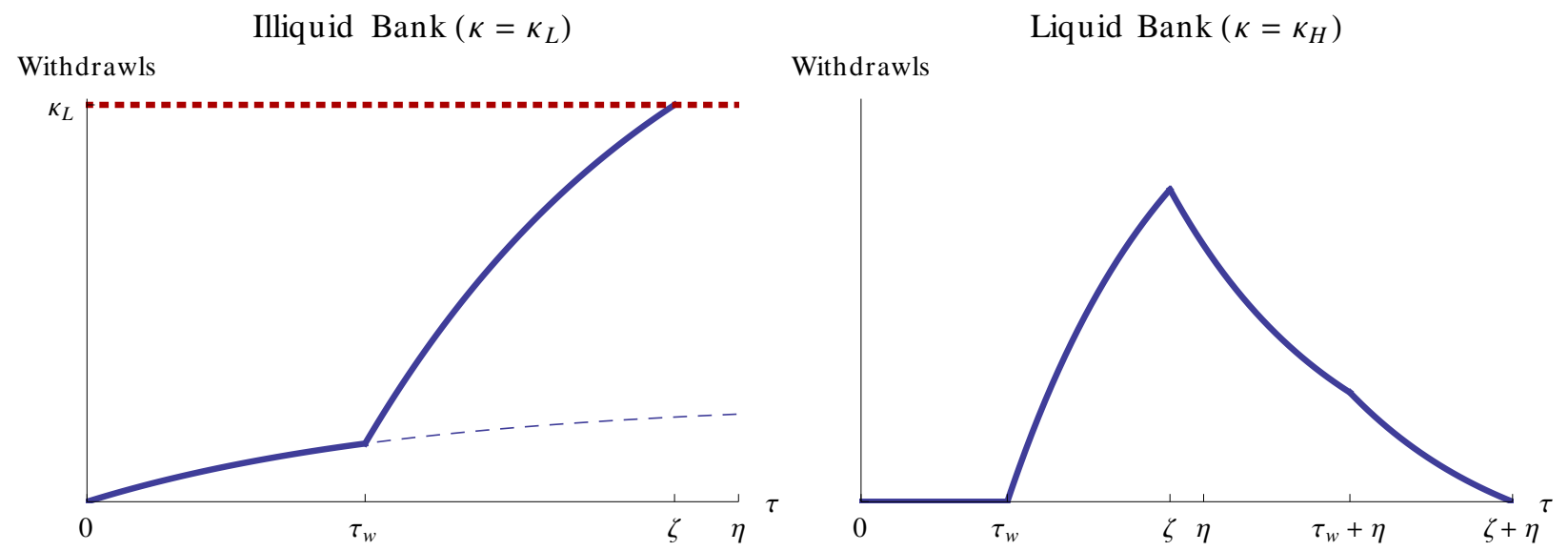

Cumulative withdrawal patterns for an illiquid bank with capacity $\kappa_{L}$ and a liquid bank with capacity $\kappa_{H}>1$. At $\tau=0$ the rumor starts to spread. Agents with a $y_{M}$ signal wait in equilibrium $\tau_{w}=0.85$ before withdrawing. The illiquid bank fails while the liquid bank starts experiencing redeposits at $\zeta=1.8$. Parameter values are $r=0.09, \beta=1, \theta=1.03, \eta=2, p_{0}=0.8, \delta=0.12, k=10^{-6}, \kappa_{L}=0.65, \alpha=0.7$, $\gamma=0.75$.

at $t_{0}+\zeta$ total withdrawals by $y_{L}$ agents are

$$
q \int_{t_{0}}^{t_{0}+\zeta} \beta U\left(t_{i} \mid t_{0}\right) d t_{i}=q\left(1-e^{-\beta \zeta}\right)
$$

Agents with $y_{M}$ signals wait for $\tau_{w}$, and at $t_{0}+\zeta$ their total withdrawals are

$$
(1-q) \int_{t_{0}}^{t_{0}+\zeta-\tau_{w}} \beta U\left(t_{i} \mid t_{0}\right) d t_{i}=(1-q)\left(1-e^{-\beta\left(\zeta-\tau_{w}\right)}\right)
$$

Figure 3 depicts the cumulative withdrawal patterns for both banks. For illiquid banks, $y_{L}$ agents begin to withdraw first right after the liquidity event $t_{0}$ as the rumor starts spreading. At $t_{0}+\tau_{w}$ to be determined shortly in equilibrium, agents with $y_{M}$ signals join the force of withdrawals, until eventually cumulative withdrawals reach $\kappa_{L}$ at $t_{0}+\zeta$.

For liquid banks, things are different. No agents are withdrawing immediately after $t_{0}$, but $y_{M}$ agents start withdrawing at $t_{0}+\tau_{w}$. At $t_{0}+\zeta$ those early informed $y_{M}$ agents realize the bank is liquid and start redepositing, which makes aggregate withdrawals decrease over time. In this range, interestingly, at the same time late informed agents are still withdrawing while early informed agents who learned that the bank is liquid begin redepositing. The intriguing empirical pattern of simultaneous withdrawing-depositing is unique to our model with rumor-based bank 
runs, and we wait for future more detailed data sets to test this implication.

\subsection{Bank Run Equilibrium Given $q$}

\subsubsection{Two-step Procedure}

Define $\tau_{r} \equiv \zeta-\tau_{w}$ as the time period that $y_{M}$ agents stay outside the bank. The label " $r$ " captures the "remaining survival time" when they decide to stay out of the bank. The bank run equilibrium given $q$ is determined in a straight-forward two-step procedure. Define $G\left(\tau_{r}\right) \equiv g\left(\zeta-\tau_{r}\right)$, i.e., replace $\zeta-\tau$ by $\tau_{r}$ in (20). From the individual $y_{M}$ agent's optimal withdraw condition in (21), the equilibrium redepositing time $\tau_{r}^{*}$ must satisfy

$$
G\left(\tau_{r}^{*}\right)=\frac{(\lambda(1-\gamma)-r) e^{\lambda \tau_{r}^{*}} p_{0}-\left(1-p_{0}\right)\left(e^{\lambda \eta}-1\right) \frac{r(r-k \delta)}{\delta-r} e^{-\delta \tau_{r}^{*}}+r\left(1-e^{\lambda \eta}\left(1-p_{0}\right)\right)}{\left(1-p_{0}\right)\left(e^{\lambda \eta}-1\right)+\left(e^{\lambda \tau_{r}^{*}}-1\right) p_{0}}=0
$$

One important observation emerge. In (25), the equilibrium survival time $\tau_{r}^{*}$ is uniquely determined, without using other endogenous variables $q$ or $\zeta$. This is because in the agent's first-order condition regarding optimal withdrawal, both the hazard rate $h\left(\tau_{w}\right)$ in $(9)$ and the continuation value $\widehat{V}_{O}\left(\tau_{w}\right)$ in (19) only depend on the remaining survival time $\tau_{r}=\zeta-\tau_{w}$.

Once we pin down $\tau_{r}^{*}$ from (25), the equilibrium survival time $\zeta^{*}$ follows from the aggregate withdrawal condition. Combing (23) and (24), the illiquid bank fails when

$$
\kappa_{L}=(1-q)\left(1-e^{-\beta \tau_{r}}\right)+q\left(1-e^{-\beta \zeta}\right)
$$

Interestingly, the remaining survival time $\tau_{r}$ also determines the cumulative withdrawals by $y_{M}$ agents. We can now solve for the equilibrium survival time $\zeta^{*}$ as

$$
\zeta^{*}=-\frac{1}{\beta} \log \left[1-\frac{\kappa_{L}-(1-q)\left(1-e^{-\beta \tau_{r}^{*}}\right)}{q}\right]
$$

\subsubsection{Why $\zeta^{*} \leq \eta$ Holds Generically?}

We next show that in our model generically the illiquid bank fails before the rumor stops spreading in the equilibrium, i.e., $\zeta^{*} \leq \eta$. In fact, our previous analysis relies on this assumption. When we wrote down the aggregate failure condition (23), we implicitly assume that at the failure time 
$t_{0}+\zeta^{*}$ there are still newly informed $y_{L}$ agents withdrawing, which exactly requires that the illiquid bank fails before the rumor stops spreading, i.e., $\zeta^{*} \leq \eta$.

A natural question follows: Is it possible to have $\zeta^{*}>\eta$ in our equilibrium? The answer is negative generically, ${ }^{14}$ unless $\zeta^{*}=\infty$ so that there is no bank run. The following lemma states this result formally.

Lemma 1. Generically, the equilibrium bank survival time $\zeta^{*}$, if finite, cannot exceed the awareness window $\eta$, so that $\zeta^{*} \leq \eta$.

Intuitively, we have seen that (25), which is the individual $y_{M}$ agent's first-order condition, pins down the equilibrium remaining survival time $\tau_{r}^{*}$ based on primitives. On the other hand, from (24), the asynchronous timing structure implies that the remaining survival time $\tau_{r}^{*}=\zeta^{*}-\tau_{w}$ also determines the cumulative withdrawal of $y_{M}$ agents to run down the illiquid bank. Therefore we need an extra degree of freedom to ensure that the remaining survival time derived from the individual $y_{M}$ agent's optimality condition is consistent with the remaining survival time required to run down the bank.

It is clear that in the above two-step procedure, the presence of $y_{L}$ agents who withdraw immediately after hearing the rumor offers such flexibility. Because the required $y_{M}$ withdrawal is the gap between the capacity $\kappa_{L}$ and the cumulative $y_{L}$ withdrawal $q\left(1-e^{-\beta \zeta}\right)$, we can find such $\zeta$ so that $y_{M}$ agents' individual incentives to withdraw coincide with the required aggregate $y_{M}$ withdrawal. Note that this argument also implies the crucial role that heterogeneous agents play in generating the interior bank run equilibrium, which becomes more clear when we discuss the equilibrium mechanism in the next subsection.

\subsubsection{Equilibrium Mechanism}

Based on the aggregate bank failure condition, we can determine natural bounds for $y_{M}$ agents' remaining survival time time $\tau_{r}^{*}$ in equilibrium. When $y_{M}$ agents withdraw immediately as $y_{L}$ agents, i.e., $\tau_{w}=0$, the distance from the $y_{M}$ agent's withdrawing time to potential bank failure,

\footnotetext{
${ }^{14}$ Here, "a statement holds generically" means that this statement may not hold only for some parameter set which is zero measure. This convention comes from the general equilibrium literature.
} 
i.e., the remaining survival time $\zeta-\tau_{w}$, will assume its upper bound value:

$$
\tau_{r}^{u}=\frac{1}{\beta} \ln \frac{1}{1-\kappa_{L}}<\eta
$$

On the other hand, $\zeta \leq \eta$ in the above lemma gives the lower bound of $\tau_{r}^{*}$ :

$$
\tau_{r}^{l}=\frac{1}{\beta} \ln \left(\frac{1-q}{1-\kappa_{L}-q e^{-\beta \eta}}\right) .
$$

Because of the parameter condition (12), $\tau_{r}^{l}>0$ so that $y_{M}$ withdrawals are also contributing to the bank's failure. These bounds are helpful in understanding the economic mechanism that pins down the equilibrium.

Intuitively, $G\left(\tau_{r}\right)$ in $(25)$ gives the sign of marginal cost minus the marginal benefit if the $y_{M}$ agent waits a bit longer. For illustration, start with the hypothetical situation where all $y_{M}$ agents withdraw immediately after hearing the rumor (i.e., $\tau_{w}=0$ ). Then remaining survival time $\tau_{r}=\zeta-\tau_{w}$ takes its upper bound $\tau_{r}^{u}$. Then, if $G\left(\tau_{r}^{u}\right)<0$ so that the marginal benefit of waiting exceeds the marginal cost, then it implies that each individual $y_{M}$ agent wants to postpone his withdrawal.

Now suppose that every $y_{M}$ agent decides to wait $\tau_{w}>0$. Importantly, there are more agents with $y_{L}$ signal withdrawing before $y_{M}$ agents, and bank failure requires less cumulative mass of withdrawing $y_{M}$ agents. This implies that the remaining survival time $\tau_{r}=\zeta-\tau_{w}$ goes down (check (26)). Thus, the closer bank failure leads to a higher failure hazard rate (check (9)), and the marginal cost of waiting goes up. To the extreme that all $y_{M}$ agents are waiting for sufficiently long, then the survival time takes the upper bound $\eta$ and the remaining survival time attains the lower bound $\tau_{r}=\tau_{r}^{l}$. Then $G\left(\tau_{r}^{l}\right)>0$ says each individual $y_{M}$ agent wants to withdraw a bit earlier. Therefore at some equilibrium withdrawal time $\tau_{w}^{*}$ we have $G\left(\tau_{r}^{*}=\zeta^{*}-\tau_{w}^{*}\right)=0$ to satisfy the individual optimality condition. We have the following proposition to summarize this discussion.

Proposition 4. Given q, the bank run equilibrium is characterized by follows:

1. If $G\left(\tau_{r}^{l}\right) \leq 0$, then there does not exist a bank run equilibrium.

2. If $G\left(\tau_{r}^{u}\right) \geq 0$, then the unique bank run equilibrium is $\tau_{r}^{*}=\zeta^{*}=\tau_{r}^{u}$ and $\tau_{w}^{*}=0$. 
3. Otherwise, we must have $G\left(\tau_{r}^{u}\right)<0$ and $G\left(\tau_{r}^{l}\right)>0$, and there exists a unique bank run equilibrium $\tau_{r}^{*} \in\left(\tau_{r}^{l}, \tau_{r}^{u}\right)$ so that $G\left(\tau_{r}^{*}\right)=0$, and

$$
\zeta^{*}=\zeta\left(\tau_{r}^{*}\right)=\frac{1}{\beta} \ln \left[\frac{q}{1-\kappa_{L}-(1-q) e^{-\beta \tau_{r}^{*}}}\right]
$$

and $\tau_{w}^{*}=\zeta\left(\tau_{r}^{*}\right)-\tau_{r}^{*}$. The equilibrium is stable.

Before moving to the next section, we briefly discuss the possibility that $y_{L}$ agents wait for some positive time. Interestingly, the argument similar to Lemma 1 implies that this cannot occur generically. If $y_{L}$ agents set a positive waiting time $\tau_{w}^{L}>0$, then it is obvious that $y_{M}$ agents will set $\tau_{w}^{M}>\tau_{w}^{L}>0$. Importantly, their respective redepositing times $\left(\tau_{r}^{M}\right.$ and $\left.\tau_{r}^{L}\right)$ have to satisfy the individual optimality conditions in the nature of (25). However, we require that these $\tau_{r}^{M}$ and $\tau_{r}^{L}$ satisfy the aggregate withdrawal condition as well, which generically cannot hold. ${ }^{15}$

\subsection{Endogenous Information Acquisition}

In our model, each informed agent endogenously acquires information about the bank's liquidity because of the potential bank failure. Therefore we first characterize the individual agent's optimal information acquisition condition, given the bank run equilibrium. On the other hand, we have seen that information quality $q$ also affects the bank run equilibrium. This intricate feedback effect between bank runs and information acquisition generally leads to two equilibria: one equilibrium where agents do not acquire information and also do not run, while the other equilibrium where agents acquire information aggressively and also run on the illiquid bank.

\subsubsection{The First-Order Condition of Information Acquisition}

We next characterize the optimal information choice. Recall that by setting $q$, we have

$$
\operatorname{Pr}\left\{y=y_{L} \mid t_{i}\right\}=q p\left(t_{i} \mid t_{i}\right), \operatorname{Pr}\left\{y=y_{M} \mid t_{i}\right\}=1-q \text {, and } \operatorname{Pr}\left\{y=y_{H} \mid t_{i}\right\}=q\left(1-p\left(t_{i} \mid t_{i}\right)\right) .
$$

The information cost is $\chi(q)=\frac{\alpha}{2} q^{2}$, which is increasing and convex in the information quality $q$.

\footnotetext{
${ }^{15}$ The aggregate withdrawal condition becomes $\kappa_{L}=(1-q)\left(1-e^{-\beta \tau_{r}^{M}}\right)+q\left(1-e^{-\beta \tau_{r}^{L}}\right)$ if both agents are withdrawing at $\zeta$. If $y_{L}$ agents already stopped, then $\kappa_{L}=(1-q)\left(1-e^{-\beta \tau_{r}^{M}}\right)+q\left(1-e^{-\beta \eta}\right)$ and we get back to the same situation as discussed in the main text.
} 
Denote the signal with quality $q$ as $y^{q}$. When agent $t_{i}$ hears the rumor he spends his information collection effort to maximize the following object, taking the equilibrium survival time $\zeta^{*}$ as given:

$$
\begin{aligned}
v(0) & =\operatorname{Pr}\left\{y=y_{L} \mid t_{i}\right\} V_{I}\left(0 \mid y_{L}\right)+\operatorname{Pr}\left\{y=y_{M} \mid t_{i}\right\} V_{I}\left(0 \mid y_{M}\right)+\operatorname{Pr}\left\{y=y_{H} \mid t_{i}\right\} V_{I}\left(0 \mid y_{H}\right)-\chi(q) \\
& =q p\left(t_{i} \mid t_{i}\right)+q\left(1-p\left(t_{i} \mid t_{i}\right)\right) \frac{\delta}{\delta-r}+(1-q) V_{I}\left(0 \mid y_{M}\right)-\chi(q)
\end{aligned}
$$

where we have used the result in Proposition 3 which also provides an expression for $V_{I}\left(0 \mid y_{M}\right)$. Note that we are implicitly taking the bank run equilibrium here, so that the agent with $y_{L}$ finds immediate withdrawal to be optimal.

Therefore, take the first order condition for $v(0)$ with respect to $q$, the optimal $q^{*}$ satisfies (recall that parameter restriction $(12))^{16}$

$$
\underbrace{p\left(t_{i} \mid t_{i}\right)+\left(1-p\left(t_{i} \mid t_{i}\right)\right) \frac{\delta}{\delta-r}}_{\mathbb{E}\left[V_{I}(0) \text { [informative signal }\right]}-\underbrace{V_{I}\left(0 \mid y_{M}\right)}_{\mathbb{E}\left[V_{I}(0) \mid \text { uninformative signal }\right]}-\alpha q^{*} \geqslant 0, \text { with equality if } q^{*}<\frac{\kappa_{L}}{1-e^{-\beta \eta}}
$$

This expression is intuitive. Raising $q$ increases (lowers) the probability of (un)informative signals, but costs more.

Combining with the dependence of $\zeta^{*}$ on $q^{*}$ in Proposition 4 and the individual agent's optimal information acquisition condition (31), we can solve for the endogenous information acquisition $q^{*}$ and survival time $\zeta^{*}$ simultaneously.

\subsubsection{Run and No-Run Equilibria}

We show that with endogenous information acquisition, in general multiple equilibria emerge. First, we check whether $q^{*}=0$ is an equilibrium. Since the marginal cost of acquiring information is zero, in order for $q^{*}=0$ to hold in equilibrium, it must be that there is no bank run. ${ }^{17}$ Of course, no bank run is also sufficient for not acquiring additional information $q^{*}=0$. According to Proposition

\footnotetext{
${ }^{16}$ The fact that we are focusing on bank run equilibrium and that the agent with $y_{L}$ finds immediate withdrawal to be optimal also imply that in (31) $q^{*}$ cannot bind at zero, as the Blackwell information theorem implies that $p\left(t_{i} \mid t_{i}\right)+\left(1-p\left(t_{i} \mid t_{i}\right)\right) \frac{\delta}{\delta-r}-V_{I}\left(0 \mid y_{M}\right)>0$ always, i.e., information has positive value as it improves the agent's decision. On the other hand, the equilibrium without bank run must have $q^{*}=0$ and is analyzed in Section 4.3.2.

${ }^{17}$ Otherwise, with bank run, immediate withdraw with $y_{L}$ signals implies a positive value for the signal. See related argument in footnote 16.
} 
4, the condition for existence of a no-run equilibrium in this case is

$$
\left.G\left(\tau_{r}^{l}(q)\right)\right|_{q=0} \leq 0
$$

When (32) fails, i.e., bank run occurs even fixing $q=0$ exogenously, then bank runs with positive information acquisition must exist. The next lemma summarizes this result.

Lemma 2. Condition (32) is the necessary and sufficient condition for the existence of an equilibrium where no bank run occurs (and therefore $q^{*}=0$ ). If (32) fails, there exist bank run equilibria with positive information acquisition $q^{*}>0$.

The above lemma only provides sufficient conditions for the existence of bank run equilibria. Interestingly, bank run equilibrium (with $q^{*}>0$ ) could exist even when (32) holds. Intuitively, although no-run-no-acquisition is an equilibrium, run-acquisition might also be an equilibrium. Once every agents raise the equilibrium $q^{*}$ above zero, a bank run is possible, and this makes individual information acquisition self-enforcing.

In general, even among the class of bank run equilibria with positive endogenous information acquisition, multiplicity may occur. The next lemma shows that under certain sufficient condition provided in the Appendix, we will have at most one such equilibrium. Essentially, this condition, by guaranteeing that at the equilibrium $q^{*}>0$ the marginal benefit of information acquisition has to go below the marginal cost of information acquisition for $q$ slightly above $q^{*}$, implies that the resulting equilibria (if exist) must be unique and stable.

Lemma 3. Under condition (39) provided in Appendix, the bank-run equilibrium with positive endogenous information acquisition, if one exists, is unique and stable.

The next proposition follows from the two above lemmas.

Proposition 5. Under (32) and the condition in Lemma 3, we have at most two equilibria. The first is no-run equilibrium without information acquisition, i.e., $q^{*}=0$. The other is the bank run equilibrium with information acquisition so that $q^{*}>0$ and $\zeta^{*}<\eta$.

From now on, to facilitate analysis, we will assume that conditions imposed in Proposition 5 hold, so that we have at most two equilibria: one with active information acquisition and bank 
Figure 4: Survival Time and Information Acquisition Response to Information Cost
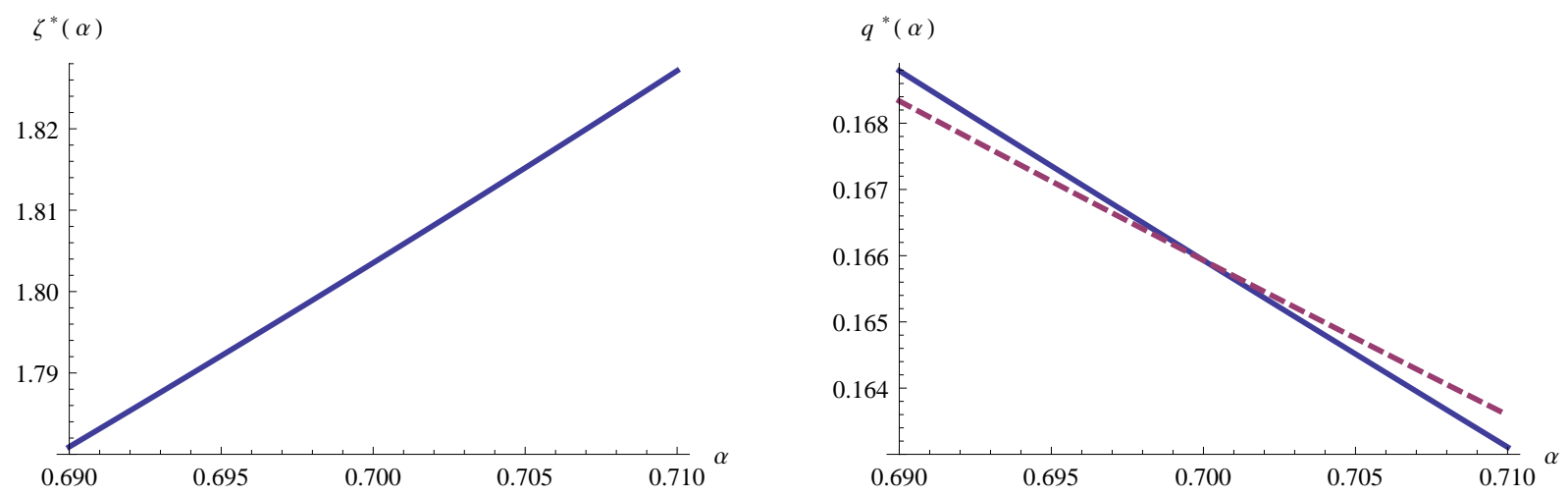

Solid lines show the equilibrium survival time of the illiquid bank $\zeta^{*}$, and the information choice $q^{*}$ as a function of the cost of information $\alpha$. The dashed line allows the equilibrium $q^{*}$ to change but holds fixed $\zeta^{*}$ at its baseline level. Parameter values are $r=0.09, \beta=1, \theta=1.03, \eta=2, p_{0}=0.8, \delta=0.12, k=10^{-6}$, $\kappa_{L}=0.65, \alpha=0.7, \gamma=0.75$.

runs, and a second without information acquisition and no run. In particular, we will be interested in parameters that eliminate the bank run equilibrium.

\subsection{Comparative Statics}

We perform comparative static analysis in this section. The following analysis focuses on the run equilibrium with endogenous information acquisition.

\subsubsection{Information Acquisition Cost $\alpha$}

When each agent finds it easier to acquire information, naturally the precision $q^{*}$ increases (the right panel in Figure 4). As shown in the left panel in Figure 4, this further leads to a shorter survival of the illiquid bank, i.e., $\zeta^{*}$ becomes lower. A lower $\zeta^{*}$ motivates each agent to learn even more aggressively, and this feedback mechanism amplifies the initial effect of a lower $\alpha$. The dashed line in Figure 4 graphs the endogenous information quality $q^{*}$ by fixing the survival time at its baseline level. When $\alpha$ drops by one percent from the baseline level 0.7 to 0.693 , the bank failure feedback mechanism leads agents to acquire 1.2 percent more information than otherwise.

This effect suggests information acquisition complementarities among depositors. Hellwig and Veldkamp (2009) show that information acquisition exhibits complementarity if and only if the actions are complementary. In our model, although running on the bank is complementary, se- 
Figure 5: Rumor Spreading Rate $\beta$ and Awareness Window $\eta$
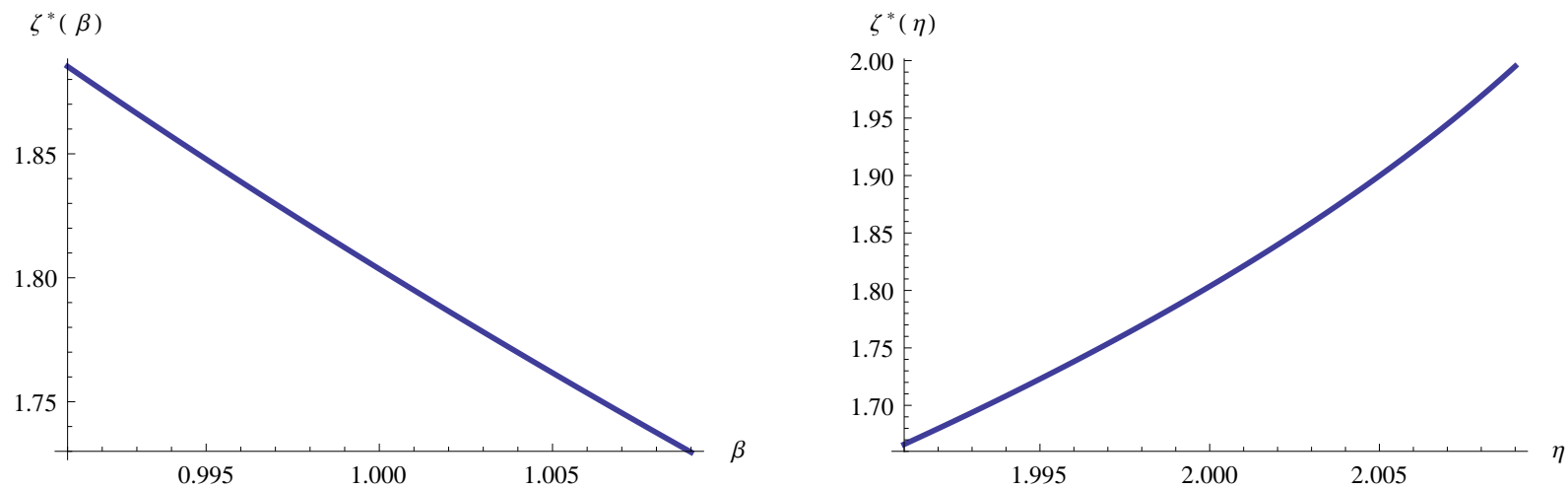

Solid lines show the equilibrium survival time of the illiquid bank $\zeta^{*}$ as a function of the rumor spreading rate $\beta$ and the length of the awareness window $\eta$. Parameter values are $r=0.09, \beta=1, \theta=1.03, \eta=2$, $p_{0}=0.8, \delta=0.12, k=10^{-6}, \kappa_{L}=0.65, \alpha=0.7, \gamma=0.75$.

quential information acquisitions do not necessarily exhibit complementarity. The substitutability naturally arises in the sequential learning setting through the dependence of the posterior belief on the survival time of the bank, which is analyzed in (33). When other depositors acquire more information, the survival time $\zeta^{*}$ is shorter. Then, the mere survival of the bank coveys more information about the bank's liquidity so that upon hearing the rumor agents perceive the bank to be stronger. Substitutability naturally arises because this effect discourages each individual depositor's motivation for information acquisition.

\subsubsection{Rumor Spreading Rate $\beta$ and Awareness Window $\eta$}

When the rumor spreading rate $\beta$ increases, all else equal, the illiquid bank will fail faster. This effect is illustrated in Figure 5. In response, each individual agent acquires more information, and the illiquid bank fails even faster. The feedback effects as discussed before are also present here, and this result is intuitive.

Relative to Abreu and Brunnermeier (2003), our model decouples the rumor spreading rate from the awareness window. When we turn to the effect of the awareness window $\eta$ on the equilibrium survival time $\zeta^{*}$ and information precision $q^{*}$, a surprising result emerges. When $\eta$ increases so that everybody knows that potentially there will be more informed agents attacking the bank, in our model each individual agent acquires information less aggressively and the illiquid bank survives longer. 
It turns out that this surprising result not only comes from our decoupling of these two effects, but also relies on the novel uncertainty structure that we introduce in this framework. The intuition can be understood by investigating the posterior probability that the bank is illiquid upon hearing the rumor and observing that the bank is still alive:

$$
p\left(t_{i} \mid t_{i}\right)=\frac{\operatorname{Pr}\left\{\text { illiquid bank survives at } t_{i} \mid \kappa_{L}, t_{i}\right\} \operatorname{Pr}\left\{\kappa_{L}\right\}}{\operatorname{Pr}\left\{\text { illiquid bank survives at } t_{i} \mid \kappa_{L}, t_{i}\right\} \operatorname{Pr}\left(\kappa_{L}\right)+\operatorname{Pr}\left\{\kappa_{H}\right\}}=\frac{\frac{e^{\lambda \zeta}-1}{e^{\lambda \eta}-1} p_{0}}{\frac{e^{\lambda \zeta}-1}{e^{\lambda \eta}-1} p_{0}+1-p_{0}}
$$

When $\eta$ is large, $t_{0} \in\left[t_{i}-\eta, t_{i}\right]$ could occur a long time ago, and the probability

$$
\operatorname{Pr}\left\{\text { illiquid bank survives at } t_{i} \mid \kappa_{L}, t_{i}\right\}=\frac{e^{\lambda \zeta}-1}{e^{\lambda \eta}-1}
$$

is lower. Consequently, conditional on the bank being alive at $t_{i}$, the bank is more likely to be liquid. Without uncertainty $\left(p_{0}=0\right.$ or 1$), p\left(t_{i} \mid t_{i}\right)$ does not depend on the awareness window $\eta$.

This interesting result differs from that of static models where usually a greater pool of proneto-run agents leads to more severe runs. Clearly, the casual intuition that runs are more severe with more prone-to-run agents comes from the argument that bank failure requires a sufficient mass of running depositors. However, our result shows that when cumulative informed agents are enough to run down the bank, artificially shortening the awareness window will gives rise to a novel information effect with the exact opposite direction.

\section{$5 \quad$ Policy Analysis and Extensions}

How much cash reserves should banks hold to prevent runs? In this section we analyze this major question, of concern to both banks and their regulators. We then extend our base model. First, we introduce fundamentally insolvent banks, so that it is also socially efficient for individual agents to acquire information. We then consider a two-bank economy where competition amplifies the individual agent's socially wasteful information production. For both extensions we discuss government policy in the recent crisis. 
Figure 6: Minimal Capacity Required to Eliminate Runs
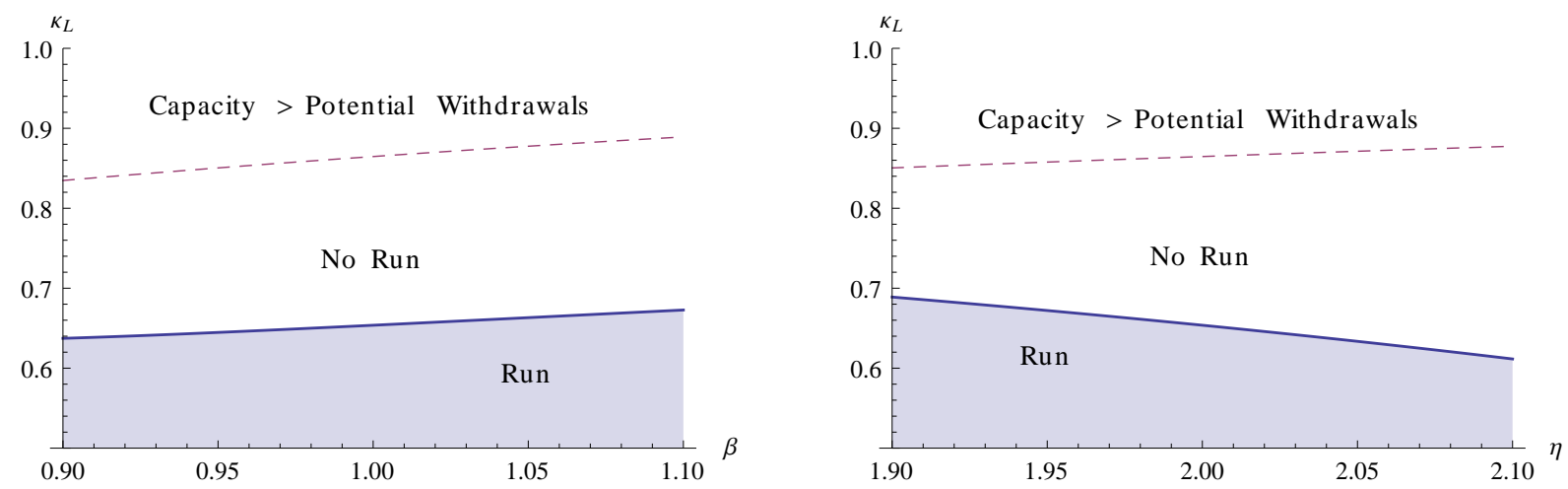

The solid line is the minimal illiquid bank capacity $\kappa_{L}$ that eliminates the bank run equilibrium as a function of the rumor spreading rate $\beta$ and the length of the awareness window $\eta$. The dashed line is the potential mass of informed agents as in (11). Parameter values are $r=0.09, \beta=1, \theta=1.03, \eta=2, p_{0}=0.8$, $\delta=0.12, k=10^{-6}, \alpha=0.7, \gamma=0.75$.

\subsection{Minimum Reserve to Eliminate Bank Runs}

In Proposition 5 we have shown that similar to static Diamond and Dybvig setting, generally in our model there are two equilibria: one equilibrium without learning and bank runs, and the other with active learning and bank runs. However, unlike the typical static setting where runs occur if the bank reserve is below all potential withdrawals, our rich dynamic setting gives non-trivial reserve threshold that eliminates the dynamic run equilibrium. In other words, due to uncertainty about the bank illiquidity and other depositors' withdrawal timings, the minimum reserve requirement that will fence off the bank run equilibrium with endogenous learning might be far below the level that is sufficient to cover all potential withdrawals.

In Figure 6, we plot the minimum reserve level $\kappa_{L}$ so that the bank run equilibrium does not exist. We plot the threshold $\kappa_{L}$ as a function of $\widehat{\beta}$ (the left panel) and $\widehat{\eta}$ (the right panel). Intuitively, when rumors spread faster (higher $\beta$ ) the bank is more susceptible to runs. The reason is that there will be more informed agents at any point in time who can potentially run on the bank. The figure shows that a faster spreading rumor requires higher cash reserves $\kappa_{L}$ to eliminate the run equilibrium. On the other hand, as discussed above, increasing the length of the awareness window $\eta$ has the counter-intuitive effect of increasing the equilibrium survival time through its effect on $p\left(t_{i} \mid t_{i}\right)$. For this reason, when the awareness window is larger, smaller cash reserves are required to prevent bank runs. 
In Figure 6 we also show that the minimal liquidity level to eliminate the run is much lower than the potential mass of informed agents. From the view of static Diamond and Dybvig runs, the potential mass of informed agents is the relevant liquidity reserve required to eliminate runs. However, due to the asynchronous nature of our rumor-based setting, even without information acquisition, immediate withdrawal after hearing the rumor might not be an equilibrium as the bank may survive for a while. Thus, eliminating dynamic rumor-based runs requires much less liquidity reserve than the one suggested by the static perspective.

One potential criticism to the above discussion is that we have so far only focused on responsive equilibria, i.e. only informed agents will run on the bank. Interestingly, in our dynamic setting with uncertainty, we can rule out one class of synchronized sun-spot type pure-strategy bank run equilibrium (a la Diamond and Dybvig), where all agents (including the uninformed) run at some arbitrary but fixed time $t^{R} \geq 0 .{ }^{18}$ First, suppose to the contrary that everyone runs at some $t^{R}>0$, which must be because the bank has some strictly positive probability being illiquid at $t^{R}$ (otherwise waiting is optimal). But if the bank is indeed potentially illiquid at $t^{R}$, then every agent would like to preempt. The reason is simple: conditional on the bank being illiquid, running at $t^{R}$ (with others) gives the agent $\kappa_{L}+\gamma\left(1-\kappa_{L}\right)<1$, while he will receive his entire deposit if he preempts the run and withdraws at $t^{R}-\epsilon$ for some $\epsilon>0$. This argument implies that the only possible synchronized run occurs at $t^{R}=0$. However, at $t^{R}=0$, it is common knowledge that the bank is liquid almost surely, as the bank starts liquid and only becomes illiquid at some liquidity event $t_{0}>0$ (recall Section 2.1.3), i.e. with probability 1 , at $t^{R}=0$ we have and $\kappa=\kappa_{H}>1$. Thus, even if everyone else withdraws immediately, an individual agent would remain in the bank and this behavior is not an equilibrium.

Finally, it is clear that for the model to generate quantitatively meaningful policy recommendations on banks' capital reserve requirement, one needs reasonable parameters to begin with. As a first step toward this goal, our stylized model falls short on this dimension. However, though beyond the scope of the current paper, given the right data, a calibration (or even estimation) of our model can certainly achieve this more ambitious goal.

\footnotetext{
${ }^{18}$ It is beyond the scope of this paper to study the interaction between our responsive equilibrium where only informed agents run gradually and this class of sunspot equilibrium where everyone runs.
} 


\subsubsection{Policy Implication: Subprime Mortgage Crisis}

On May 17, 2007 Fed Chairman Bernanke indicated in a speech about the subprime mortgage market that looser lending standards where pervasive especially in loans originated in 2006 (Bernanke, 2007). The speech took place at a time when low teaser rates on these adjustable-rate mortgages were set to expire, suggesting that the rise in defaults was just the tip of an iceberg. Subsequently, money market funds experienced the modern-day equivalent of a bank "run" as asset-backed commercial paper outstanding dropped from $\$ 1.3$ trillion in July 2007 to $\$ 833$ billion in December 2007 Acharya, Schnabl, and Suarez (2011).

One interpretation within our model of this speech is that it signaled that the awareness window was relatively short, in which case there was not much to learn from the survival of the money market to that point in time. Suppose that instead, the analysis revealed that looser lending standards were pervasive in loans originated since 2003. In that case, information about the resulting weakness must have been spreading for a long time. Having observed that the money market kept growing despite this fact, investors would conclude that the probability of the system being liquid enough is high and a run might not occur.

\subsection{Insolvent Banks and Stress Tests}

So far our model focuses on runs on fundamentally solvent but potentially illiquid banks. Because the first best allocation is keeping the bank alive always, information is socially "bad." Of course, in practice information may be "good" because of the presence of fundamentally insolvent banks so that runs on them are socially efficient. To address this issue, we introduce insolvency into our model. We then argue that the planner may mitigate running on fundamentally solvent banks by helping individual agents spot those insolvent banks more easily through stress tests.

\subsubsection{Setup}

Suppose that after the liquidity event that occurs at $t_{0}$, the bank in our model might be insolvent, which randomly fails (rather than matures) with intensity $\xi>r$. In this event deposits recover 0 for each dollar, and therefore $\xi>r$ implies that the bank is indeed insolvent. Of course the bank can also be solvent, and if so it can be either liquid or illiquid as we modeled before. 
Naturally, there will be two layers of information. The first layer of information is regarding the bank's solvency which is both socially and individually valuable. The second is information regarding the bank's liquidity condition, which is individually valuable (and socially destructive) when agents realize that runs on the illiquid bank become a concern. We assume that although these two layers of information are different, they are inevitably related when individual agents are acquiring them. As shown shortly, this way we endogenize individuals endowed liquidity information precision via the active information collection about bank solvency.

Now when agent $t_{i}$ hears the rumor, the possibility of insolvency motivates him to spend a fixed amount of effort $\underline{e}>0$ to obtain a signal $\mathbf{1}_{z} \in\{0,1\}$; for simplicity, this signal $\mathbf{1}_{z}$ perfectly reveals whether the bank is solvent or not. ${ }^{19}$ We focus on situations where, in equilibrium, the agent always finds it optimal to acquire this solvency signal (which is guaranteed by a sufficiently high default intensity $\xi$ ). Therefore, conditional on the bank being insolvent, all agents who hear the rumor know that the bank is insolvent and therefore run on the bank immediately. ${ }^{20}$

A by-product of the agent's private learning about the bank solvency is that he also learns something about the liquidity of the bank. Given the effort $\underline{e}$ of figuring out whether the bank is insolvent, if the bank turns out to be solvent, then the baseline quality of the bank's liquidity signal $y$ - which is the signal we modeled in Section 2.1.4 - is just $\underline{e}$. As a result, the agent's additional liquidity information precision choice is $q \geq \underline{e}$ with acquisition cost $\frac{\alpha}{2}(q-\underline{e})^{2}$. The interpretation is that the process of collecting insolvency information inevitably teaches the agent more about the bank's liquidity. The longer the process of collecting insolvency information, the more the agent knows about the bank's liquidity. Our modeling that the effort of collecting insolvency information (i.e., $\underline{e}$ ) becomes the baseline quality of liquidity information captures this idea in the simplest way.

\subsubsection{Policy Implication: Stress Tests}

The above setting has important implications for stress tests in revealing the fundamentally problematic banks. By providing insolvency information only, the government can use stress tests to reduce $\underline{e}$ to eliminate runs on solvent-but-illiquid banks.

According to our model, if a great effort is required to learn about insolvent banks (say Lehman),

\footnotetext{
${ }^{19}$ This assumption is for clarifying the economic channel and innocuous. See detailed discussion in footnote 21.

${ }^{20}$ Suppose that the insolvent bank has a capacity of $\kappa_{Z}$; therefore the equilibrium failure time for insolvent bank is $t_{0}+\zeta_{z}^{*}=t_{0}+\frac{1}{\beta} \ln \frac{1}{1-\kappa_{Z}}$.
} 
i.e., a higher $\underline{e}$, then each agent will be automatically endowed with significant information about the liquidity of solvent banks (say Citibank). Given that, it is more likely that bank runs exist in the equilibrium, and therefore they are more likely to acquire even more information. As a result, runs on solvent banks start.

Stress tests provide transparency on potentially insolvent banks (Bernanke, 2009), and therefore reduce $\underline{e}$. Government, by providing higher quality information about banks' insolvency, can crowd out private acquisition of insolvency information. Because public information can be better targeted at insolvency alone, while the process of private acquisition of solvency information inevitably reveals liquidity information, public provision of solvency information helps all agents know that other agents do not have superior information regarding banks liquidity situation. Therefore, our model suggests that the public provision of insolvency information indirectly reduces the socially wasteful information acquisition regarding liquidity, and therefore make runs on illiquid banks less likely.

We emphasize that the perfect revelation of insolvent bank in our setting helps clarify the channel of releasing better solvency information to help illiquid banks; more precisely, it is not through higher average bank quality once the stress test isolates those insolvent ones. ${ }^{21}$ Rather, the channel is through the fact that now everybody knows that everybody will wait to see the stress test and therefore not scramble to search the insolvent banks. As a result everybody will have less precise information on which solvent bank is less liquid and susceptible to a run.

This view is consistent with the Federal Reserve Board's recent break from the traditional supervisory view of opaqueness in favor of more public disclosure to restore the confidence of investors. However, our rationale is different than the one described by Bernanke (2010) who argues that the release of detailed information about the banks subjected the tests to scrutiny by outside analysts, thereby enhancing the credibility of the tests. Instead, we argue that by providing more information, the government crowds out the information collection effort by individuals about

\footnotetext{
${ }^{21}$ When the planner varies $\underline{e}$, agents can always perfectly spot insolvent banks, therefore the channel of insolvency information is shut down. Rather, the channel is the strategic interaction of individual agents who make their endogenous acquisition decision on liquidity information. In fact, our model can be handle the case that insolvent banks are imperfectly revealed. As discussed after Proposition 4, in our model generically a bank run equilibrium exists only when $y_{L}$ agents withdraw immediately, and the equilibrium analysis is identical if we instead assume that agents cannot perfectly tell insolvent banks from illiquid ones (so they will withdraw immediately once receiving $y_{L}$ signal or $\mathbf{1}_{z}=1$ ). However, under this alternative assumption, it is quite obvious that better solvency information helps illiquid banks since it allows individual agents to tell them apart from insolvent ones.
} 
Figure 7: Minimal Capacity and Information Collection Effort Required to Eliminate Runs

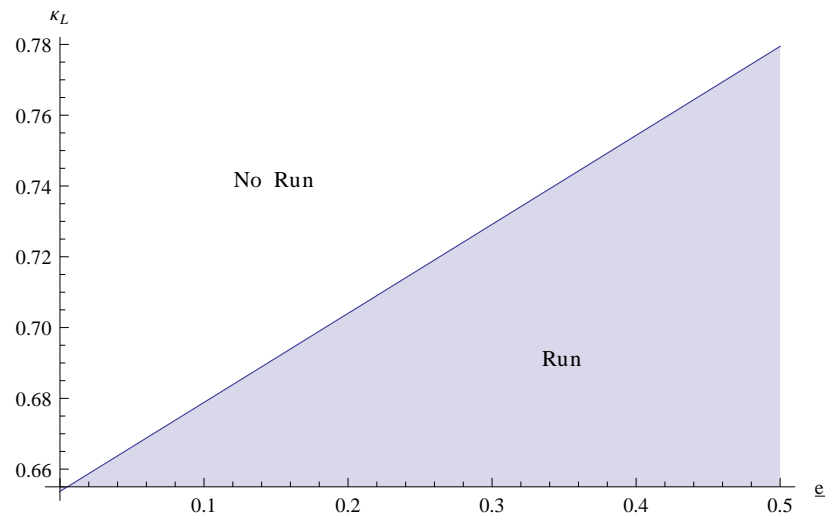

We plot the minimal illiquid bank capacity $\kappa_{L}$ that eliminates the bank run equilibrium as a function of the information collection effort $\underline{e}$. Parameter values are $r=0.09, \beta=1, \theta=1.03, \eta=2, p_{0}=0.8, \delta=0.12$, $k=10^{-6}, \kappa_{L}=0.65, \alpha=0.7, \gamma=0.75$.

the solvency of the banks. Since liquidity and solvency are tightly related, this government policy has the useful by-product of reducing information collection about bank liquidity and therefore reducing the incidence of runs on illiquid banks.

Figure 7 plots the minimal illiquid bank capacity $\kappa_{L}$ that eliminates the bank run equilibrium as a function of the information collection effort $\underline{e}$. We see from Figure 7 that as the government provides more information about the solvency of the bank (lower $\underline{e}$ ), less liquid banks (lower $\kappa_{L}$ ) can avoid runs.

\subsection{Multiple Solvent Banks and Policy Implications}

We now investigate the behavior of depositors and their incentives to acquire information in the presence of competing solvent banks. Instead of holding cash, a bank run in this setting involves the transfer of deposited funds from one bank to another.

We show that greater information quality leads agents to inefficient runs on the otherwise identical solvent bank. ${ }^{22}$ Here, the two banks' difference might be minuscule, and in fact transfers between the two institutions only involve social losses (transaction fees and bank failure). The planner thus can inject noise into the system, so that individual agents with less informative signals

\footnotetext{
${ }^{22}$ Some anecdotal evidence that differences in depositor perception about bank liquidity motivates them to withdraw from illiquid banks is provided by Sidel, Enrich, and Fitzpatrick (2008): "Melody Williams, 50 years old, said in the past 30 days she has moved about $\$ 25,000$ out of Washington Mutual, spreading it to other financial institutions she thought were stronger, including Wells Fargo \& Co. Ms. Williams, the controller for an architecture firm, said she thought that Washington Mutual had gotten 'too big for their britches' with too many deals over the years."
} 
are more likely to stay in their original bank without knowing which one is the (more) liquid one.

\subsubsection{Analysis}

Suppose there are two banks, ex-ante identical except that half the population deposits in bank $A$ and half deposits in bank $B$. Both banks promise the same rate of return $r$. However, transferring funds between banks requires a transaction cost $k$. A liquidity event occurs at a random time $t_{0}$, and the rumor starts that exactly one of the banks is illiquid $\left(0<\kappa_{L}<1\right)$ while the other is liquid $\left(\kappa_{H}>1\right)$. The prior probability that each bank is illiquid is $p_{0}=0.5$ since they are ex-ante identical. The learning process in the two bank set-up is simpler, because the passage of time without a failure teaches agents nothing about the relative viability of their bank.

As in the setup above, agents are allowed to acquire a costly signal $\widetilde{y} \in\left\{y_{L}, y_{M}, y_{H}\right\}$ about the status of their bank with probability distribution as in (2). Agents who receive the $y_{H}$ signal know that their bank is the liquid one and therefore never withdraw. Agents who receive the $y_{M}$ signal gain no useful information. Since there is a small transaction cost $k$, remaining in their original bank is optimal. ${ }^{23}$ Finally, agents who draw the $y_{L}$ signal run on their bank immediately. From their perspective, the value of a dollar in their bank falls and the value of a dollar in the competing bank increases to a riskless $\frac{\delta}{\delta-r}$. Thus, as long as the transaction cost $k$ is small enough, immediate withdraw is optimal. ${ }^{24}$ Information is (privately) more valuable in this setup since the outside option is a nearly identical bank rather then holding cash.

The following proposition characterizes the equilibrium in this setting with two banks.

Proposition 6. Under the two banks setup, the bank run equilibrium $\left\{\zeta^{*}, q^{*}\right\}$ is determined by the following two equations:

$$
\begin{gathered}
\zeta^{*}=-\frac{1}{\beta} \ln \left(1-\frac{\kappa_{L}}{q^{*}}\right), \\
\frac{1}{2}(1-k) \frac{\delta}{\delta-r}+\frac{1}{2} \frac{\delta}{\delta-r}-V_{I}\left(0 \mid y_{M} ; \zeta^{*}, q^{*}\right)=\alpha q^{*},
\end{gathered}
$$

where the expression for $V_{I}\left(0 \mid y_{M} ; \zeta^{*}, q^{*}\right)$ is given in the Appendix.

\footnotetext{
${ }^{23}$ Formally, let $V_{-I}\left(0 \mid y_{M}\right)$ be the value of a dollar in the other bank from these agents' perspective. Then $V_{-I}\left(0 \mid y_{M}\right)=(1-k) V_{I}\left(0 \mid y_{M}\right)<V_{I}\left(0 \mid y_{M}\right)$, which is the value of a dollar in the original bank.

${ }^{24}$ More specifically, we require $k$ is sufficiently small that $V_{I}\left(0 \mid y_{M}\right)<(1-k) V_{-I}\left(0 \mid y_{H}\right)=(1-k) \frac{\delta}{\delta-r}$.
} 
Figure 8: Minimal Capacity and Information Cost Required to Eliminate Runs

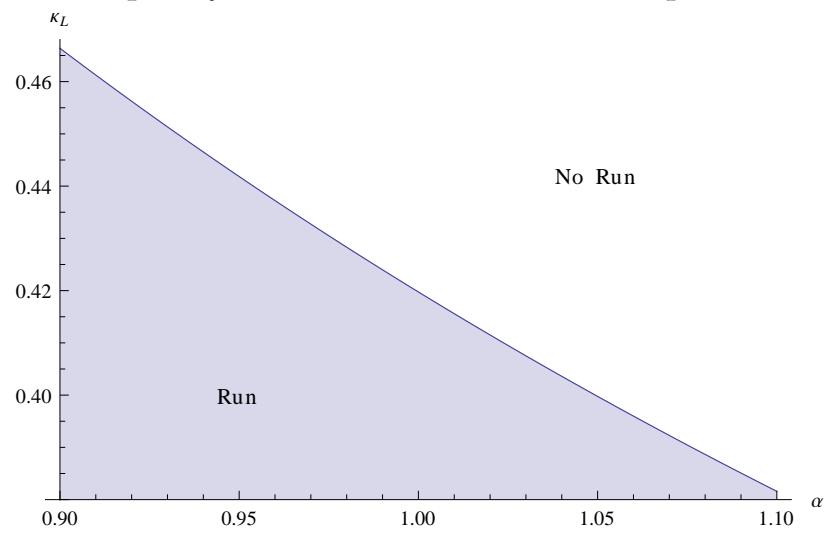

We plot the minimal illiquid bank capacity $\kappa_{L}$ that eliminates the bank run equilibrium as a function of the information cost $\alpha$ for the two bank setup. Parameter values are $r=0.09, \beta=1, \theta=1.03, \eta=2, \delta=0.12$, $k=10^{-6}, \kappa_{L}=0.3, \alpha=1, \gamma=0.75$.

\subsubsection{Injecting Noise}

In this extension, higher information quality $q$ about the liquidity of two solvent banks is socially undesirable, as it shortens the survival time of the illiquid bank by introducing more agents who realize that one bank strictly dominates the other. Injecting noise can alleviate the problem, and the simplest interpretation of injecting noise is to raise the information acquisition cost $\alpha$.

A bank run equilibrium requires that withdrawals by the $y_{L}$ agents alone can destroy a bank, i.e. $\kappa_{L}<q\left(1-e^{-\beta \eta}\right) .{ }^{25}$ The threshold $\bar{q}$ so that no run would occur is $\bar{q} \equiv \frac{\kappa_{L}}{1-e^{-\beta \eta}}$. If the planner raises $\alpha$ so that the marginal benefit of acquiring information is below its marginal cost, i.e.,

$$
\frac{1}{2}(1-k) \frac{\delta}{\delta-r}+\frac{1}{2} \frac{\delta}{\delta-r}-V_{I}\left(0 \mid y_{M}\right) \leq \alpha \bar{q}
$$

then the illiquid bank is always liquid enough to sustain a run. Figure 8 plots the minimal illiquid bank capacity $\kappa_{L}$ that eliminates the bank run equilibrium as a function of the information cost $\alpha$ for the two bank setup. The injection of noise into the economy (higher $\alpha$ ) blurs the differences between the competing solvent banks. The noise reduces the equilibrium information quality $q$, and as a result, a run is easier to eliminate and requires less reserves by the illiquid bank.

Consider the recent financial crisis in 2008. A fear that some banks were insolvent prompted the Capital Purchase Program commonly known as the bailout of the nine largest U.S. financial

\footnotetext{
${ }^{25}$ In this two-bank setting we no long impose the restriction of $q \leq \frac{\kappa_{L}}{1-e^{-\beta \eta}}$ as in condition (12), because now only $y_{L}$ agents are withdrawing to potentially take down the illiquid bank.
} 
institutions on October 13, 2008. When presenting the program to the CEOs of the 9 banks, Secretary of Treasury Henry M. Paulson was concerned that the strongest banks, e.g., JPMorgan, would not participate. ${ }^{26}$ To make sure that they do, government officials suggested that if a bank refused the funds, its regulator would later force it to raise capital anyway and under worse terms. ${ }^{27}$ The government was in fact injecting noise about the liquidity of competing solvent banks into the economy. By pooling banks together the incentive to transfer funds between them was kept low enough so that none of the nine banks suffered a run.

\section{Conclusion}

We study the endogenous information acquisition and withdrawal-redeposit decisions of individual agents when a liquidity event triggers a spreading rumor and therefore exposes a bank to a run. Uncertainty about the bank's liquidity and potential failure motivate agents who hear the rumor to acquire additional information to improve signal quality. Although the bank run equilibrium is unique given the signal's quality, multiple equilibria emerge with endogenous information acquisition activities. We show that a bank run equilibrium exists when agents aggressively acquire information, and study several tools that the government can use to prevent runs.

Learning and information acquisition are indeed considered some of the key drivers of bank runs, both of which affect government disclosure policy regarding bank regulatory information. Given the newly found popularity of stress tests both in the U.S. and in Europe, we show that public provision of insolvency information can help curb the private information acquisition effort on bank liquidity. As a result, carefully constructed stress tests can help prevent bank runs by crowding out information acquisition by individuals. The planner must be careful however to avoid providing too much information that differentiates competing solvent-but-illiquid banks from liquid ones, for such information can start a run.

Finally, the dynamic bank run model we provide above can shed new light on other economic settings such as arbitrageur behavior, currency attacks, and R\&D investment games.

\footnotetext{
26 "I was concerned about Jamie Dimon, because JPMorgan appeared to be in the best shape of the group, and I wanted to be sure he would accept the capital." - Paulson (2010)

27 "Look, we're making you an offer," I said, jumping in. "If you don't take it and sometime later your regulator tells you that you are undercapitalized and you have to raise private-sector capital but you are unable to do so, you may not like the terms if you have to come back to me." - Paulson (2010)
} 


\section{References}

Abreu, Dilip, and Markus K. Brunnermeier, 2002, Synchronization risk and delayed arbitrage, Journal of Financial Economics 66, 341 - 360.

— 2003, Bubbles and crashes, Econometrica.

Acharya, Viral, Douglas Gale, and Tanju Yorulmazer, 2011, Rollover risk and market freezes, Journal of Finance 66, 1175-1207.

Acharya, Viral V., Philipp Schnabl, and Gustavo Suarez, 2011, Securitization without risk transfer, Working Paper.

Bernanke, Ben S., 2007, The subprime mortgage market, Speech at the Federal Reserve Bank of Chicago 43rd Annual Conference on Bank Structure and Competition.

— 2009, The supervisory capital assessment program, Speech at the Federal Reserve Bank of Atlanta 2009 Financial Markets Conference, Jekyll Island, Georgia.

— 2010, The supervisory capital assessment program-one year later, Speech at the Federal Reserve Bank of Chicago 46th Annual Conference on Bank Structure and Competition, Chicago, Illinois.

Bhattacharya, Sudipto, and Douglas Gale, 1987, Preference shocks, liquidity, and central bank policy, in W. Barnett and K. Singleton eds., New Approaches to Monetary Policy, Cambridge University Press pp. 69-88.

Brunnermeier, Markus K., and John Morgan, 2010, Clock games: Theory and experiments, Games and Economic Behavior 68, 532 - 550 .

Chari, V. V., and Ravi Jagannathan, 1988, Banking panics, information, and rational expectations equilibrium, The Journal of Finance 43, pp. 749-761.

Diamond, Douglas W., and Philip H. Dybvig, 1983, Bank runs, deposit insurance, and liquidity, The Journal of Political Economy 91, pp. 401-419.

Goldstein, Itay, and Ady Pauzner, 2005, Demand-deposit contracts and the probability of bank runs, Journal of Finance 60, pp. 1293-1327.

Gorton, Gary, 1985, Bank suspension of convertibility, Journal of Monetary Economics 15, 177 193.

Green, Edward J., and Ping Lin, 2003, Implementing efficient allocations in a model of financial intermediation, Journal of Economic Theory 109, 1 - 23.

Gu, Chao, 2011, Herding and bank runs, Journal of Economic Theory 146, 163-188.

He, Zhiguo, and Wei Xiong, forthcoming 2011a, Dynamic debt runs, Review of Financial Studies.

— , forthcoming 2011b, Rollover risk and credit risk, Journal of Finance.

Hellwig, Christian, and Laura Veldkamp, 2009, Knowing what others know: Coordination motives in information acquisition, Review of Economic Studies 76, 223-251. 
Iyer, Rajkamal, and Manju Puri, forthcoming 2011, Understanding bank runs: The importance of depositor-bank relationships and networks, American Economic Review.

Jacklin, Charles J., and Sudipto Bhattacharya, 1988, Distinguishing panics and information-based bank runs: Welfare and policy implications, Journal of Political Economy 96, pp. 568-592.

Morris, Stephen, and Hyun Song Shin, 2002, Social value of public information, The American Economic Review 92, 1521-1534.

Paulson, Henry M., 2010, On the Brink: Inside the Race to Stop the Collapse of the Global Financial System (Business Plus).

Sidel, Robin, David Enrich, and Dan Fitzpatrick, 2008, WaMu is seized, sold off to J.P. Morgan, in largest failure in U.S. banking history, The Wall Street Journal p. A1.

Wallace, Neil, 1988, Another attempt to explain an illiquid banking: the diamond-dybvig model with sequential service taken seriously, Fed Reserve Bank Minneapolis Quraterly Review 12, 2-16.

\section{A Appendix A}

\section{A.1 Proof of Proposition 1}

We have two cases to consider for the hazard rate. First, suppose $\zeta>\eta$. Then an agent informed at $t_{i}$ learns nothing from the fact that the bank has not failed by $t_{i}$. His distribution of failure dates $t_{i}+\tau$ is

$$
\Pi\left(t_{i}+\tau \mid t_{i}\right)= \begin{cases}0 & \tau<\zeta-\eta \\ p_{0} \frac{e^{\lambda \eta}-e^{\lambda(\zeta-\tau)}}{e^{\lambda \eta}-1} & \zeta-\eta \leq \tau<\zeta \\ p_{0} & \zeta \leq \tau .\end{cases}
$$

with non-zero density $\pi\left(t_{i}+\tau \mid t_{i}\right)=p_{0} \frac{\lambda e^{\lambda(\zeta-\tau)}}{e^{\lambda \eta}-1}$ for $\zeta-\eta<\tau<\zeta$. On the other hand, if $\zeta \leq \eta$, then agent $t_{i}$ 's distribution of failure dates $t_{i}+\tau$ is

$$
\Pi\left(t_{i}+\tau \mid t_{i}\right)= \begin{cases}0 & \tau<0 \\ p\left(t_{i} \mid t_{i}\right) \frac{1-e^{-\lambda \tau}}{1-e^{-\lambda \zeta}} & 0 \leq \tau<\zeta \\ p\left(t_{i} \mid t_{i}\right) & \zeta \leq \tau .\end{cases}
$$

non-zero density $\pi\left(t_{i}+\tau \mid t_{i}\right)=p\left(t_{i} \mid t_{i}\right) \frac{\lambda e^{-\lambda \tau}}{1-e^{-\lambda \zeta}}$ for $0<\tau<\zeta$. Plugging either of the pairs into the definition of the hazard rate (8) yields after some algebraic manipulation the same expression (9) for any $\tau \geq \max \{0, \zeta-\eta\}$ and zero elsewhere.

\section{A.2 Proof of Proposition 2}

We first establish the following lemma.

Lemma 4. The function $g(\tau)$ crosses zero from below at most once in the interval $[0, \zeta]$. 
Proof. Since it is the change in the numerator that dominates around $g(\tau)=0$, it suffices to show that the numerator of $g(\tau)$ (ignoring the constant)

$$
(\lambda(1-\gamma)-r) e^{\lambda(\zeta-\tau)} p_{0}-\left(1-p_{0}\right)\left(e^{\lambda \eta}-1\right) \frac{r(r-k \delta)}{\delta-r} e^{-\delta(\zeta-\tau)}
$$

is increasing over the interval $[0, \zeta]$. Furthermore, since $\lambda(1-\gamma)-r<\lambda(1-\gamma)-r(1-k)<0$ from (13), it follows that (36) is concave in $\tau$. Let $\bar{\tau}$ be the unique maximizer. At the maximum

$$
\zeta-\bar{\tau}=\frac{1}{\lambda+\delta} \ln \frac{\delta\left(1-p_{0}\right)\left(e^{\lambda \eta}-1\right) \frac{r(r-k \delta)}{\delta-r}}{\lambda(r-\lambda(1-\gamma)) p_{0}}<0
$$

due to (13). Thus, the function in (36) attains its maximum to the right of $\zeta$ and is therefore increasing over $[0, \zeta]$.

Lemma 4 implies that if $g(\zeta) \leq 0(g(0) \geq 0)$ then $g(\tau)<0(g(\tau) \geq 0)$ always for $\tau \in[0, \zeta]$. We next consider the optimal strategy for the three cases of the proposition:

Case 1. If $g(\zeta) \leq 0$, then it is optimal to stay in the bank always. To prove our claim, it suffices to show that $V_{I}(\tau)>V_{O}(\tau)$ for $\tau \in[0, \zeta]$. Suppose not, then there must exist some $\tau_{w}$ so that $V_{I}\left(\tau_{w}\right)=V_{O}\left(\tau_{w}\right)$ and $V_{I}^{\prime}\left(\tau_{w}\right)>V_{O}^{\prime}\left(\tau_{w}\right)$ because $V_{I}(\zeta)>V_{O}(\zeta)$. From HJB equations, we have

$$
h\left(\tau_{w}\right)(1-\gamma)-r V_{I}\left(\tau_{w}\right)=V_{I}^{\prime}\left(\tau_{w}\right)-V_{O}^{\prime}\left(\tau_{w}\right)>0
$$

However, since $V_{I}\left(\tau_{w}\right) \geq V_{O}\left(\tau_{w}\right) \geq \widehat{V}_{O}\left(\tau_{w}\right)$ by definition (the first inequality is because there is no transaction cost to take one dollar out, and the second inequality is because $\widehat{V}_{O}\left(\tau_{w}\right)$ may be derived under suboptimal policy), we have

$$
h\left(\tau_{w}\right)(1-\gamma)-r V_{I}\left(\tau_{w}\right)<h\left(\tau_{w}\right)(1-\gamma)-r \widehat{V}_{O}\left(\tau_{w}\right)=g\left(\tau_{w}\right) \leq 0,
$$

a contradiction.

Case 2. If $g(0) \geq 0$, then it is optimal to withdraw at 0 and redeposit right after $\zeta$. Well, if $g(0) \geq 0$, then $g(\tau) \geq 0$ always for $\tau \in[0, \zeta]$, and $g(\zeta)>0$. Using $g(\zeta)>0$, we first show that since $k$ is arbitrarily small, there exists some $\widehat{\tau}$ close to $\zeta$ so that $V_{O}(\widehat{\tau})=V_{I}(\widehat{\tau})$. To show this, we show that $V_{O}^{\prime}(\zeta)-V_{I}^{\prime}(\zeta)$ is strictly below zero when $k$ is arbitrarily small. To see this, from the HJB equations we know that

$$
\begin{aligned}
V_{O}^{\prime}(\zeta)-V_{I}^{\prime}(\zeta) & =h(\zeta)\left(V_{O}(\zeta)-V_{I}(\zeta)+\gamma-1\right)+\delta\left(V_{I}(\zeta)-V_{O}(\zeta)\right)+r V_{I}(\zeta) \\
& =-g(\zeta)+(h(\zeta)+\delta-r)\left(V_{O}(\zeta)-V_{I}(\zeta)\right)
\end{aligned}
$$

The first is strictly negative while the second term converges to zero as $k \rightarrow 0$. Therefore, when $k$ is arbitrary small there exists some $\varepsilon$ so that $V_{I}(\zeta-\varepsilon)<V_{O}(\zeta-\varepsilon)$. Due to continuity and the fact that $V_{I}(\zeta)=\frac{V_{O}(\zeta)}{1-k}>V_{O}(\zeta)$, there exists some $\widehat{\tau}$ close to $\zeta$ so that $V_{I}(\widehat{\tau})=V_{O}(\widehat{\tau})$. Note that $V_{O}(\widehat{\tau})=\widehat{V}_{O}(\widehat{\tau})$. 
Now to prove that "it is optimal to withdraw at 0 and redeposit right after $\zeta$," we only need to show that $V_{I}(\tau)=V_{O}(\tau)$ holds for all $\tau \in[0, \widehat{\tau}]$ (intuitively, at any point of time a dollar inside the bank has the value of taking outside, it is always optimal to keep the money outside). Suppose that this does not hold; since $V_{I}(\tau) \geq V_{O}(\tau)$ in general, there must exits some point $\tau_{w} \in[0, \widehat{\tau}]$ so that $V_{I}\left(\tau_{w}\right)=V_{O}\left(\tau_{w}\right)$ and $V_{I}^{\prime}\left(\tau_{w}\right)<V_{O}^{\prime}\left(\tau_{w}\right)$. Choosing the largest value $\tau_{w}$, so that $V_{O}\left(\tau_{w}\right)=\widehat{V}_{O}\left(\tau_{w}\right)$ holds (i.e., the optimal continuation strategy is wait outside the bank until $\zeta$ ). Similar to the argument before, we have

$$
h\left(\tau_{w}\right)(1-\gamma)-r V_{O}\left(\tau_{w}\right)=V_{I}^{\prime}\left(\tau_{w}\right)-V_{O}^{\prime}\left(\tau_{w}\right)<0,
$$

but this contradicts the fact that $h\left(\tau_{w}\right)(1-\gamma)-r V_{O}\left(\tau_{w}\right)=h\left(\tau_{w}\right)(1-\gamma)-r \widehat{V}_{O}\left(\tau_{w}\right) \geq 0$ since $g(\tau) \geq 0$ always.

Case 3. It follows from the Lemma 4 that $g(\zeta)>0$ and $g(0)<0$ imply that there exists a unique $\tau_{w} \in(0, \zeta)$ so that $g\left(\tau_{w}\right)=0, g(\tau)>0$ for $\tau \in\left(\tau_{w}, \zeta\right)$ and $g(\tau)<0$ for $\tau \in\left(0, \tau_{w}\right)$. Following the same argument in the second part by replacing 0 with $\tau_{w}$, we know that it is optimal to withdraw at $\tau_{w}$ and redeposit at $\zeta+$, and $V_{I}\left(\tau_{w}\right)=V_{O}\left(\tau_{w}\right)=\widehat{V}_{O}\left(\tau_{w}\right)$. Then to prove our claim we only need to show that $V_{I}(\tau)>V_{O}(\tau)$ for $\tau \in\left(0, \tau_{w}\right)$.

Let $H(\tau) \equiv V_{I}(\tau)-V_{O}(\tau)$ with $H\left(\tau_{w}\right)=0$, we need to show that $H(\tau)>0$ for $\tau \in\left(0, \tau_{w}\right)$, as $H(\tau)=V_{I}(\tau)-V_{O}(\tau) \geqslant 0$ in general. First, we show that it is impossible to have $H(\tau)=0$ uniformly on any interval $\left(\tau_{w}-\Delta, \tau_{w}\right)$ where $\Delta>0$; if it is true then it must be that $V_{I}(\tau)=$ $V_{O}(\tau)=\widehat{V}_{O}(\tau)$ on that interval so that

$$
\begin{aligned}
0 & =r V_{I}(\tau)+h(\tau)\left(\gamma-V_{I}(\tau)\right)+\delta\left(1-V_{I}(\tau)\right)+V_{I}^{\prime}(\tau) \\
& =r \widehat{V}_{O}(\tau)+h(\tau)\left(\gamma-\widehat{V}_{O}(\tau)\right)+\delta\left(1-\widehat{V}_{O}(\tau)\right)+\widehat{V}_{O}^{\prime}(\tau) \\
& =r \widehat{V}_{O}(\tau)-h(\tau)(1-\gamma)=-g(\tau)>0
\end{aligned}
$$

where the first equality is (18) and third equality is using the ODE for $\widehat{V}_{O}(\tau)$ with $0=h(\tau)\left(1-\widehat{V}_{O}(\tau)\right)+$ $\delta\left(1-\widehat{V}_{O}(\tau)\right)+\widehat{V}_{O}^{\prime}(\tau)$. This contradiction implies that we must have $V_{I}(\tau)>V_{O}(\tau)$ for some $\tau$ close to $\tau_{w}$. Now suppose that there exists another point $\tau_{w}^{1}<\tau_{w}$ so that $V_{I}\left(\tau_{w}^{1}\right)=V_{O}\left(\tau_{w}^{1}\right)$. Take $\tau_{w}^{1}$ that is closet to $\tau_{w}$ so that $V_{I}^{\prime}\left(\tau_{w}^{1}\right) \geq V_{O}^{\prime}\left(\tau_{w}^{1}\right)$. At $\tau_{w}^{1}, V_{O}\left(\tau_{w}^{1}\right)$ must satisfy the HJB in (18) (because $\tau_{w}^{1}$ is in the inaction region around the neighborhood, i.e., $(1-k) V_{I}(\tau)<V_{O}(\tau)$ for $\tau$ close to $\left.\tau_{w}\right)$. Then

$$
\begin{aligned}
0 & =r V_{I}\left(\tau_{w}^{1}\right)+h\left(\tau_{w}^{1}\right)\left(\gamma-V_{I}\left(\tau_{w}^{1}\right)\right)+\delta\left(1-V_{I}\left(\tau_{w}^{1}\right)\right)+V_{I}^{\prime}\left(\tau_{w}^{1}\right) \\
& \geq r V_{O}\left(\tau_{w}^{1}\right)+h\left(\tau_{w}^{1}\right)\left(\gamma-V_{O}\left(\tau_{w}^{1}\right)\right)+\delta\left(1-V_{O}\left(\tau_{w}^{1}\right)\right)+V_{O}^{\prime}\left(\tau_{w}^{1}\right) \\
& =r V_{O}\left(\tau_{w}^{1}\right)-h\left(\tau_{w}^{1}\right)(1-\gamma) \geq-g\left(\tau_{w}^{1}\right)>0
\end{aligned}
$$

where we have used the HJB for $V_{O}\left(\tau_{w}^{1}\right)$, and the fact that $V_{O}\left(\tau_{w}^{1}\right) \geq \widehat{V}_{O}\left(\tau_{w}^{1}\right)$ in general. Again we get a contradiction with (18). 


\section{A.3 Proof of Proposition 3}

Proof. Given $\tau_{w}$ simple integration yields

$$
V_{I}\left(0 \mid y_{M}\right)=\frac{\left[\begin{array}{c}
\frac{\delta\left(e^{\lambda \eta}\left(1-p_{0}\right)-1\right)}{\delta-r}\left(1-e^{-(\delta-r) \tau_{w}}\right)+\frac{\delta+\lambda \gamma}{\lambda+\delta-r} e^{\lambda \zeta} p_{0}\left(1-e^{-(\lambda+\delta-r) \tau_{w}}\right) \\
+e^{-(\delta-r) \tau_{w}}\left(e^{\lambda \eta}-1-\left(e^{\lambda \eta}-e^{\lambda\left(\zeta-\tau_{w}\right)}\right) p_{0}\right) V_{O}\left(\tau_{w}\right)
\end{array}\right]}{e^{\lambda \eta}-1-e^{\lambda \eta} p_{0}+e^{\lambda \zeta} p_{0}}
$$

Note that $h\left(\tau_{w}\right)(1-\gamma)=r V_{O}\left(\tau_{w}\right)$ implies that

$$
\left(e^{\lambda \eta}-1-\left(e^{\lambda \eta}-e^{\lambda\left(\zeta-\tau_{w}\right)}\right) p_{0}\right) V_{O}\left(\tau_{w}\right)=\frac{\lambda(1-\gamma) e^{\lambda\left(\zeta-\tau_{w}\right)} p_{0}}{r}
$$

where we used the definition of $h$ in (8). Then note that

$$
\frac{\lambda(1-\gamma)}{r}-\frac{\delta+\lambda \gamma}{\lambda+\delta-r}=\frac{(\lambda+\delta)(\lambda(1-\gamma)-r)}{r(\lambda+\delta-r)}
$$

which gives our expression.

\section{A.4 Proof of Lemma 1}

Proof. Suppose that $\zeta>\eta$, so that at $\zeta$ the cumulative withdrawal from $y_{L}$ agents is $q\left(1-e^{-\beta \eta}\right)$. Then using the aggregate condition (26), we can back out the equilibrium $\tau_{r}$ for $y_{M}$ agents as

$$
\tau_{r}=-\frac{1}{\beta} \ln \left(1-\frac{\kappa_{L}-q\left(1-e^{-\beta \eta}\right)}{1-q}\right) .
$$

However, unless parameters are such that the above $\tau_{r}$ happens to satisfy $G\left(\tau_{r}\right)=0$ which is the $y_{M}$ agents' optimal waiting decision, generically this cannot occur.

\section{A.5 Proof of Proposition 4}

First note that the $G$ function is the mirror image of individual FOC condition function, i.e., $G\left(\tau_{r}\right)=g\left(\zeta-\tau_{r}\right)$, and it shares the same (but opposite) property of $g(\cdot)$ shown in Lemma 4:

Corollary 1. $G\left(\tau_{r}\right)$ crosses zero from above at most once on $\tau_{r} \in[0, \zeta]$.

This result implies that the following holds for the three cases of the proposition: 
Case 1. If $G\left(\tau_{r}^{l}\right) \leq 0$, then $G\left(\tau_{r}\right) \leq 0$ for all $\tau_{r} \geq \tau_{r}^{l}$. Thus if all other agents strategy is to redeposit after any $\tau_{r} \geq \tau_{r}^{l}$, it is optimal for the individual agent to deviate and wait a bit longer. Therefore, $\zeta^{*} \rightarrow \infty$ and no run equilibrium exists.

Case 2. If $G\left(\tau_{r}^{u}\right) \geq 0$, then $G\left(\tau_{r}\right) \geq 0$ for all $\tau_{r} \leq \tau_{r}^{u}$. Thus, if all other agents' strategy is to withdraw at some interior $\tau_{r} \leq \tau_{r}^{u}$, it is optimal for the individual agent to deviate and withdraw earlier. Therefore, agents withdraw immediately in the only symmetric equilibrium.

Case 3. Finally, if $G\left(\tau_{r}^{u}\right)<0$ and $G\left(\tau_{r}^{l}\right)>0$ then by continuity of $G$ and Corollary 1 , there exists a unique bank run equilibrium $\tau_{r}^{*} \in\left(\tau_{r}^{l}, \tau_{r}^{u}\right)$ so that $G\left(\tau_{r}^{*}\right)=0$. Plugging into (26) we get the equilibrium survival time $\zeta^{*}$ and waiting time $\tau_{w}^{*}$. A second implication of Corollary 1 is that $G^{\prime}\left(\tau_{r}^{*}\right)<0$. Therefore the equilibrium is stable.

\section{A.6 Proof of Lemma 2}

Proof. The proof of the first statement is given in the text. To show the second statement, we need to show that the agent's FOC in information acquisition

$$
A(q) \equiv p\left(t_{i} \mid t_{i}\right)+\left(1-p\left(t_{i} \mid t_{i}\right)\right) \frac{\delta}{\delta-r}-V_{I}\left(0 \mid y_{M}\right)-\alpha q \geqslant 0 \text { with equality if } q<\frac{\kappa_{L}}{1-e^{-\beta \eta}},
$$

combined with bank-run equilibrium condition, has a solution. Note that both $p\left(t_{i} \mid t_{i}\right)$ and $V_{I}\left(0 \mid y_{M}\right)$ (given in (33) and Proposition 3) depend on $\zeta$ that is determined in Proposition 4 about bank run equilibrium given $q$. Now because (32) fails, the bank run equilibrium exists even with exogenously given $q=0$. From (22) it is optimal to withdraw given $y_{L}$. The agent's strategy depends on his signal, and therefore information has a positive value, i.e. $A(0)=p\left(t_{i} \mid t_{i}\right)+\left(1-p\left(t_{i} \mid t_{i}\right)\right) \frac{\delta}{\delta-r}-$ $V_{I}\left(0 \mid y_{M}\right)>0$. Now suppose that $q$ takes its upper bound $\frac{\kappa_{L}}{1-e^{-\beta \eta}}$; if $A\left(q=\frac{\kappa_{L}}{1-e^{-\beta \eta}}\right)>0$ then the upper bound information quality and associated bank run equilibrium is just the equilibrium that we are after. If instead $A\left(q=\frac{\kappa_{L}}{1-e^{-\beta \eta}}\right)<0$, then an interior equilibrium exists because of the continuity of $A(q)$.

\section{A.7 Proof of Lemma 3}

Proof. First, when the bank run equilibrium occurs with corner solution $\zeta=\tau_{r}^{u}=\frac{1}{\beta} \ln \frac{1}{1-\kappa_{L}}$, then the marginal benefit of information $M B=p\left(t_{i} \mid t_{i}\right)+\left(1-p\left(t_{i} \mid t_{i}\right)\right) \frac{\delta}{\delta-r}-V_{I}\left(0 \mid y_{M}\right)$ is independent 
of $q$, and the equilibrium $q^{*}$ equates $M C=\alpha q^{*}=M B$. Therefore the equilibrium is unique and stable ( $M B$ is constant while $M C$ increases with $q)$. Also if $q^{*}$ takes the upper bound corner value, the associated run equilibrium is unique as well. So the rest of proof focus on the case where both the information quality of equilibrium survival time take interior solutions.

From now on we focus on interior bank run equilibrium. Importantly, this implies that $\tau_{r}$ is determined in (25) which only depends on primitives. Therefore we treat $\tau_{r}$ as a primitive parameter. The FOC (31) when the agent sets $q^{*}$ is

$$
\frac{\left[\begin{array}{c}
\left(e^{\lambda \zeta}-1\right) p_{0}+\left(1-p_{0}\right)\left(e^{\lambda \eta}-1\right) \frac{\delta}{\delta-r}-\frac{\delta\left(e^{\lambda \eta}\left(1-p_{0}\right)-1\right)}{\delta-r}\left(1-e^{-(\delta-r) \tau_{w}}\right) \\
-\frac{\delta+\lambda \gamma}{\lambda+\delta-r} e^{\lambda \zeta} p_{0}-e^{-(\delta-r) \tau_{w}} e^{\lambda \tau_{r}} p_{0}\left(\frac{(\lambda+\delta)(\lambda(1-\gamma)-r)}{r(\lambda+\delta-r)}\right)-\alpha q\left(\left(1-p_{0}\right)\left(e^{\lambda \eta}-1\right)+\left(e^{\lambda \zeta}-1\right) p_{0}\right)
\end{array}\right]}{\left(1-p_{0}\right)\left(e^{\lambda \eta}-1\right)+\left(e^{\lambda \zeta}-1\right) p_{0}}=0
$$

where $\zeta$ and $\tau_{w}=\zeta-\tau_{r}$ depend on $q$ through (27). We have

$$
\zeta^{\prime}(q)=\tau_{w}^{\prime}(q)=\frac{e^{-\beta \zeta}-e^{-\beta \tau_{r}}}{q \beta e^{-\beta \zeta}}=\frac{1-e^{\beta \tau_{w}}}{q \beta}<0 .
$$

The derivative at the point where (38) takes zero value yields:

$$
\begin{aligned}
& \lambda \zeta^{\prime} e^{\lambda \zeta} p_{0}-\delta\left(e^{\lambda \eta}\left(1-p_{0}\right)-1\right) e^{-(\delta-r) \tau_{w}} \tau_{w}^{\prime}-\frac{\delta+\lambda \gamma}{\lambda+\delta-r} p_{0} \lambda e^{\lambda \zeta} \zeta^{\prime}+(\delta-r) e^{-(\delta-r) \tau_{w}} e^{\lambda \tau_{r}} p_{0}\left(\frac{(\lambda+\delta)(\lambda(1-\gamma)-r)}{r(\lambda+\delta-r)}\right) \tau_{w}^{\prime} \\
& -\alpha\left(\left(1-p_{0}\right)\left(e^{\lambda \eta}-1\right)+\left(e^{\lambda \zeta}-1\right) p_{0}\right)-\alpha q e^{\lambda \zeta} \lambda \zeta^{\prime} p_{0} \\
& =\lambda \zeta^{\prime} e^{\lambda \zeta} p_{0}\left[\frac{\lambda(1-\gamma)-r}{\lambda+\delta-r}-\alpha q\right]+e^{-(\delta-r)\left(\zeta-\tau_{r}\right)} \tau_{w}^{\prime}\left[e^{\lambda \tau_{r}} p_{0}(\delta-r)\left(\frac{(\lambda+\delta)(\lambda(1-\gamma)-r)}{r(\lambda+\delta-r)}\right)-\delta\left(e^{\lambda \eta}\left(1-p_{0}\right)-1\right)\right] \\
& -\alpha\left(\left(1-p_{0}\right)\left(e^{\lambda \eta}-1\right)+\left(e^{\lambda \zeta}-1\right) p_{0}\right) \\
& =\zeta^{\prime}(q) e^{-(\delta-r) \zeta}\left[\lambda e^{(\lambda+\delta-r) \zeta} p_{0}\left[\frac{\lambda(1-\gamma)-r}{\lambda+\delta-r}-\alpha q\right]+e^{(\delta-r) \tau_{r}}\left[e^{\lambda \tau_{r}} p_{0}(\delta-r)\left(\frac{(\lambda+\delta)(\lambda(1-\gamma)-r)}{r(\lambda+\delta-r)}\right)-\delta\left(e^{\lambda \eta}\left(1-p_{0}\right)-1\right)\right]\right] \\
& -\alpha\left(\left(1-p_{0}\right)\left(e^{\lambda \eta}-1\right)+\left(e^{\lambda \zeta}-1\right) p_{0}\right)
\end{aligned}
$$

The second line is clearly negative. Assume that (note that $e^{\lambda \eta}\left(1-p_{0}\right)<1$ )

$$
\begin{aligned}
& e^{(\delta-r) \tau_{r}}\left[e^{\lambda \tau_{r}} p_{0}(\delta-r)\left(\frac{(\lambda+\delta)(\lambda(1-\gamma)-r)}{r(\lambda+\delta-r)}\right)-\delta\left(e^{\lambda \eta}\left(1-p_{0}\right)-1\right)\right] \\
+ & \lambda e^{(\lambda+\delta-r) \eta} p_{0}\left[\frac{\lambda(1-\gamma)-r}{\lambda+\delta-r}-\alpha \frac{\kappa_{L}}{1-e^{-\beta \eta}}\right]>0 .
\end{aligned}
$$

Then, since $\zeta<\eta, q^{*}<\frac{\kappa_{L}}{1-e^{-\beta \eta}}$, and $\zeta^{\prime}(q)<0$, the first line is also negative. As a result, the derivative of (38) is always negative. As a result, when (38) equals zero, it must go down. Combined with differentiability of (38), this result rules out multiple solutions, because if there exist, then there must have one solution with the local slope being nonnegative. Therefore, (38) crosses zero at most once from above, which implies that the bank run equilibrium, if exists, is unique and stable. 


\section{A.8 Proof of Proposition 5}

Proof. Now suppose that $q^{*}=\frac{\kappa_{L}}{1-e^{-\beta \eta}}$ which is the upper bound, so that $\tau_{r}^{l}=0$. Suppose that

$$
(\lambda(1-\gamma)-r) p_{0}-\left(1-p_{0}\right)\left(e^{\lambda \eta}-1\right) \frac{r(r-k \delta)}{\delta-r}+r\left(1-e^{\lambda \eta}\left(1-p_{0}\right)\right) \geq 0
$$

so that run equilibrium occurs. There must be another smallest $\underline{q}$ so that run could occur. The lower bound $\underline{q}$ satisfies $G\left(\tau_{r}^{l}(\underline{q})\right)=0$, i.e., $\underline{q}$ solves

$$
\begin{gathered}
(\lambda(1-\gamma)-r)\left(\frac{1-\underline{q}}{1-\kappa_{L}-\underline{q} e^{-\beta \eta}}\right)^{\frac{\lambda}{\beta}} p_{0}- \\
\left(1-p_{0}\right)\left(e^{\lambda \eta}-1\right) \frac{r(r-k \delta)}{\delta-r}\left(\frac{1-\underline{q}}{1-\kappa_{L}-\underline{q} e^{-\beta \eta}}\right)^{-\frac{\delta}{\beta}}+r\left(1-e^{\lambda \eta}\left(1-p_{0}\right)\right)=0 .
\end{gathered}
$$

At this $\underline{q}, \zeta=\eta$ and $\tau_{w}=\eta-\frac{1}{\beta} \ln \left(\frac{1-\underline{q}}{1-\kappa_{L}-\underline{q} e^{-\beta \eta}}\right)$, and we need to check if agents has sufficient incentives to improve signal quality (note that in this case $p\left(t_{i} \mid t_{i}\right)=p_{0}$ because $\zeta=\eta$ )

$$
p_{0}+\left(1-p_{0}\right) \frac{\delta}{\delta-r}-V_{I}\left(0 \mid y_{M}\right)>\alpha q^{*}
$$

If this is true, then we will have another point $q^{*}>\underline{q}$ to be the bank run equilibrium. Otherwise, each individual agent would like to lower their information $q$ below $q$ - but we know lower $q$ cannot trigger bank run, contradiction. Moreover, it implies that at $\underline{q}, \overline{F O C}=M B-M C<0$. Given the following conditions, FOC has to goes down once it touches zero, so there will be no $q>\underline{q}$ to generate bank run equilibrium. The FOC in (31) is

$$
\frac{\left[\begin{array}{c}
\left(e^{\lambda \zeta}-1\right) p_{0}+\left(1-p_{0}\right)\left(e^{\lambda \eta}-1\right) \frac{\delta}{\delta-r}-\frac{\delta\left(e^{\lambda \eta}\left(1-p_{0}\right)-1\right.}{\delta-r}\left(1-e^{-(\delta-r) \tau_{w}}\right) \\
-\frac{\delta+\lambda \gamma}{\lambda+\delta-r} e^{\lambda \zeta} p_{0}-e^{-(\delta-r) \tau_{w}} e^{\lambda \tau_{r}} p_{0}\left(\frac{(\lambda+\delta)(\lambda(1-\gamma)-r)}{r(\lambda+\delta-r)}\right)-\alpha q^{*}\left(\left(1-p_{0}\right)\left(e^{\lambda \eta}-1\right)+\left(e^{\lambda \zeta}-1\right) p_{0}\right)
\end{array}\right]}{\left(1-p_{0}\right)\left(e^{\lambda \eta}-1\right)+\left(e^{\lambda \zeta}-1\right) p_{0}}
$$

we know that that $\tau_{r}$ is independent of $q$, and

$$
\begin{aligned}
\zeta & \in\left[\frac{1}{\beta} \ln \frac{1}{1-\kappa_{L}}, \eta\right] \\
\tau_{w} & \in\left[0, \eta-\tau_{r}\right]
\end{aligned}
$$

and

$$
\zeta^{\prime}(q)=\tau_{w}^{\prime}(q)=\frac{e^{-\beta \zeta}-e^{-\beta \tau_{r}}}{q \beta e^{-\beta \zeta}}=\frac{1-e^{\beta \tau_{w}}}{q \beta}>\frac{1-e^{\beta\left(\eta-\tau_{r}\right)}}{\underline{q} \beta}
$$


derivative at $\mathrm{FOC}=0$

$$
\begin{aligned}
& \lambda \zeta^{\prime} e^{\lambda \zeta} p_{0}-\delta\left(e^{\lambda \eta}\left(1-p_{0}\right)-1\right) e^{-(\delta-r) \tau_{w}} \tau_{w}^{\prime}-\frac{\delta+\lambda \gamma}{\lambda+\delta-r} p_{0} \lambda e^{\lambda \zeta} \zeta^{\prime}+(\delta-r) e^{-(\delta-r) \tau_{w}} e^{\lambda \tau_{r}} p_{0}\left(\frac{(\lambda+\delta)(\lambda(1-\gamma)-r)}{r(\lambda+\delta-r)}\right) \tau_{w}^{\prime} \\
& -\alpha\left(\left(1-p_{0}\right)\left(e^{\lambda \eta}-1\right)+\left(e^{\lambda \zeta}-1\right) p_{0}\right)-\alpha q^{*} e^{\lambda \zeta} \lambda \zeta^{\prime} p_{0} \\
= & \lambda \zeta^{\prime} e^{\lambda \zeta} p_{0}\left[\frac{\lambda(1-\gamma)-r}{\lambda+\delta-r}-\alpha q^{*}\right]+e^{-(\delta-r) \tau_{w}} \tau_{w}^{\prime}\left[e^{\lambda \tau_{r}} p_{0}(\delta-r)\left(\frac{(\lambda+\delta)(\lambda(1-\gamma)-r)}{r(\lambda+\delta-r)}\right)-\delta\left(e^{\lambda \eta}\left(1-p_{0}\right)-1\right)\right] \\
& -\alpha\left(\left(1-p_{0}\right)\left(e^{\lambda \eta}-1\right)+\left(e^{\lambda \zeta}-1\right) p_{0}\right) \\
= & \zeta^{\prime}(q)\left[\lambda e^{\lambda \zeta} p_{0}\left[\frac{\lambda(1-\gamma)-r}{\lambda+\delta-r}-\alpha q^{*}\right]+e^{-(\delta-r) \tau_{w}}\left[e^{\lambda \tau_{r}} p_{0}(\delta-r)\left(\frac{(\lambda+\delta)(\lambda(1-\gamma)-r)}{r(\lambda+\delta-r)}\right)-\delta\left(e^{\lambda \eta}\left(1-p_{0}\right)-1\right)\right]\right] \\
& -\alpha\left(\left(1-p_{0}\right)\left(e^{\lambda \eta}-1\right)+\left(e^{\lambda \zeta}-1\right) p_{0}\right)
\end{aligned}
$$

Assume that (note that $e^{\lambda \eta}\left(1-p_{0}\right)<1$ )

$$
e^{\lambda \tau_{r}} p_{0}(\delta-r)\left(\frac{(\lambda+\delta)(\lambda(1-\gamma)-r)}{r(\lambda+\delta-r)}\right)-\delta\left(e^{\lambda \eta}\left(1-p_{0}\right)-1\right)>0
$$

Now we put bounds on each term. we have

$$
0>\lambda e^{\lambda \zeta} p_{0}\left[\frac{\lambda(1-\gamma)-r}{\lambda+\delta-r}-\alpha q^{*}\right]>\lambda e^{\lambda \eta} p_{0}\left[\frac{\lambda(1-\gamma)-r}{\lambda+\delta-r}-\alpha\right]
$$

and

$$
\begin{gathered}
e^{-(\delta-r) \tau_{w}}\left[e^{\lambda \tau_{d}} p_{0}(\delta-r)\left(\frac{(\lambda+\delta)(\lambda(1-\gamma)-r)}{r(\lambda+\delta-r)}\right)-\delta\left(e^{\lambda \eta}\left(1-p_{0}\right)-1\right)\right] \\
>e^{-(\delta-r)\left(\eta-\tau_{d}\right)}\left[e^{\lambda \tau_{d}} p_{0}(\delta-r)\left(\frac{(\lambda+\delta)(\lambda(1-\gamma)-r)}{r(\lambda+\delta-r)}\right)-\delta\left(e^{\lambda \eta}\left(1-p_{0}\right)-1\right)\right]
\end{gathered}
$$

and finally the last term is

$$
-\alpha\left(\left(1-p_{0}\right)\left(e^{\lambda \eta}-1\right)+\left(e^{\lambda \zeta}-1\right) p_{0}\right)<-\alpha\left(\left(1-p_{0}\right)\left(e^{\lambda \eta}-1\right)+\left(e^{\lambda \frac{1}{\beta} \ln \frac{1}{1-\kappa_{L}}}-1\right) p_{0}\right)
$$

therefore the FOC are bounded above by

$$
\begin{aligned}
& \frac{1-e^{\beta\left(\eta-\tau_{d}\right)}}{\underline{q} \beta}\left\{\lambda e^{\lambda \eta} p_{0}\left[\frac{\lambda(1-\gamma)-r}{\lambda+\delta-r}-\alpha\right]+e^{-(\delta-r)\left(\eta-\tau_{d}\right)}\left[(\delta-r) \frac{\lambda(1-\gamma)}{r} e^{\lambda \tau_{d}} p_{0}-\delta\left(e^{\lambda \eta}\left(1-p_{0}\right)-1\right)-\frac{\delta+\lambda \gamma}{\lambda+\delta-r} p_{0}(\delta-r) e^{\lambda \tau_{d}}\right]\right\} \\
& -\alpha\left(\left(1-p_{0}\right)\left(e^{\lambda \eta}-1\right)+\left(e^{\lambda \frac{1}{\beta} \ln \frac{1}{1-\kappa_{L}}}-1\right) p_{0}\right)
\end{aligned}
$$

if this quantity is negative, then there is only one interior solution.

\section{A.9 Proof of Proposition 6}

Proof. At the time of hearing the rumor, the value of a dollar in the bank for agents with $y_{L}$ and $y_{H}$ signals are respectively $V_{I}\left(0 \mid y_{L}\right)=\frac{(1-k) \delta}{\delta-r}$ and $V_{I}\left(0 \mid y_{H}\right)=\frac{\delta}{\delta-r}$. In order to calculate the value for agents with the $y_{M}$ signal, note that with probability $1 / 2$ the original bank is the illiquid one, but the agent can deposit his funds (after the liquidation cost $1-\gamma$ ) to the liquid one. As a result, 
the value with $y_{M}$ signal is

$$
\begin{aligned}
V_{I}\left(0 \mid y_{M}\right) & =\frac{1}{2} \frac{1}{1-\Pi\left(0 \mid \kappa_{L}\right)} \int_{0}^{\zeta}\left[\delta e^{-(\delta-r) s}\left(1-\Pi\left(s \mid \kappa_{L}\right)\right)+e^{-(\delta-r) s} \pi\left(s \mid \kappa_{L}\right) \gamma(1-k) \frac{\delta}{\delta-r}\right] d s+\frac{1}{2} \frac{\delta}{\delta-r} \\
& =\frac{1}{2} \int_{0}^{\zeta}\left[\delta e^{-(\delta-r) s}\left(1-\frac{1-e^{-\lambda s}}{1-e^{-\lambda \zeta}}\right)+e^{-(\delta-r) s} \frac{\lambda e^{-\lambda s}}{1-e^{-\lambda \zeta}} \gamma(1-k) \frac{\delta}{\delta-r}\right] d s+\frac{1}{2} \frac{\delta}{\delta-r} \\
& =\frac{1}{2} \frac{1}{1-e^{-\lambda \zeta}} \int_{0}^{\zeta}\left[-e^{-\lambda \zeta} \delta e^{-(\delta-r) s}+\delta e^{-(\delta-r+\lambda) s}+\lambda \gamma(1-k) \frac{\delta}{\delta-r} e^{-(\delta-r+\lambda) s}\right] d s+\frac{1}{2} \frac{\delta}{\delta-r} \\
& =\frac{1}{2} \frac{1}{1-e^{-\lambda \zeta}}\left[-e^{-\lambda \zeta} \frac{\delta}{\delta-r}\left(1-e^{-(\delta-r) \zeta}\right)+\frac{\delta}{\delta-r+\lambda}\left(1-e^{-(\delta-r+\lambda) \zeta}\right)+\frac{\lambda \gamma(1-k)}{\delta-r+\lambda} \frac{\delta}{\delta-r}\left(1-e^{-(\delta-r+\lambda) \zeta}\right)\right]+\frac{1}{2} \frac{\delta}{\delta-r} \\
& =\frac{1}{2} \frac{1}{1-e^{-\lambda \zeta}}\left[-e^{-\lambda \zeta} \frac{\delta}{\delta-r}\left(1-e^{-(\delta-r) \zeta}\right)+\frac{\delta}{\delta-r+\lambda}\left(1+\frac{\lambda \gamma(1-k)}{\delta-r}\right)\left(1-e^{-(\delta-r+\lambda) \zeta)]+\frac{1}{2} \frac{\delta}{\delta-r}} .\right.\right.
\end{aligned}
$$

\section{B Appendix B}

We consider the non-stationary part of the model here. If $t_{0}<\eta$, then some early informed agents with $t_{i}<\eta$ knows that $t_{0} \in\left[0, t_{i}\right]$, and this truncation implies strategy may be $t_{i}$-dependent. However, as shown in Abreu and Brunnermeier (2003), those early agents will be bunching together to eliminate the non-stationarity. We modify their results to our setting.

Focus on the bank being illiquid. To be precise, follow Abreu and Brunnermeier (2003) in our model the agents who hears the rumor before $\zeta-\tau_{w}=\tau_{r}$ will behave as if the agent who hears the rumor exactly at $\tau_{r}$. The strategy of the agent who hears rumor at $\tau_{r}$ is that, independent of signal $\left(y_{L}\right.$ or $\left.y_{M}\right)$ he will withdraw at $\tau_{r}+\tau_{w}=\zeta$. Moreover, for agents who hear rumor at $t_{i} \in\left[\tau_{r}, \zeta\right]$, they take the following strategy. If they receive $y_{L}$ signal then he will withdraw at $\zeta$, while if they get $y_{M}$ signal then they withdraw at $t_{i}+\tau_{w}$. This additional modification is because relative to Abreu and Brunnermeier (2003) agents may have different signals in our model.

For illustration, suppose that $t_{0}=0$ so the bank should fail at $\zeta$. Recall that there are $q$ measure of $y_{L}$ signals and $1-q$ measure of $y_{M}$ signals. Since the information keeps spreading at $\zeta$ (recall that $\eta<\zeta)$ and all agents hears the rumor before $\zeta$ will withdraw at $\zeta$, there are $q\left(1-e^{-\beta \zeta}\right)$ measure of $y_{L}$ agents withdrawing. On the other hand, $y_{M}$ agents who hear the rumor in the interval $\left[0, \tau_{r}\right]$ are withdrawing at $\zeta$, with a total mass of $(1-q)\left(1-e^{-\beta \tau_{r}}\right)$. Therefore, we have

$$
(1-q)\left(1-e^{-\beta \tau_{r}}\right)+q\left(1-e^{-\beta \zeta}\right)=\kappa_{L},
$$

which is exactly (26). A similar argument can be applied to the case of $t_{0}>0$ so that the bank failure time is $t_{0}+\zeta$ : this is because endogenously there are less agents bunching at the physical time $\zeta$, so the bank failure time is postponed to $t_{0}+\zeta>\zeta$.

There is one issue that our richer (than Abreu and Brunnermeier (2003)) setting leads to potential non-stationarity. Although withdraw behavior can be stationary, the endogenous learning about bank liquidity is non-stationary when $\eta<\zeta$. In fact, initially when $t_{0}=0$, agents have no other information so $p\left(t_{i} \mid t_{i}\right)=p_{0}$ must holds. In stationary state, $p\left(t_{i} \mid t_{i}\right)=\widehat{p}_{0} \equiv \frac{\left(e^{\lambda \zeta}-1\right) p_{0}}{\left(1-p_{0}\right)\left(e^{\lambda \eta}-1\right)+\left(e^{\lambda \zeta}-1\right) p_{0}}<$ $p_{0}$. This difference potentially alters the optimal withdraw strategies for agents with different 
timings. To resolve this issue, we simply assume that for $t_{i}<\eta$, the prior is time-varying

$$
p_{0}\left(t_{i}\right)=\frac{\left(e^{\lambda t_{i}}-1\right) p_{0}}{\left[\left(1-p_{0}\right)\left(e^{\lambda \eta}-1\right)+p_{0}\left(e^{\lambda t_{i}}-1\right)\right]},
$$

and one can show that with this specification, the resulting posterior upon hearing the rumor, $p\left(t_{i} \mid t_{i}\right)$, is always $\widehat{p}_{0}$. One can presumably achieve this by a more structural way; for instance, introduce other shocks so that, conditional on survival and hearing the rumor, the posterior of the bank being illiquid is always $\widehat{p}_{0}$. Also, we have to fix the signal quality structure the $q$ in (2) is the same as in the stationary phase. We deem these technical issue non-essential for the economic questions that we are after in this paper. 